\title{
Numerical Exploration of Kaldorian Macrodynamics: Enhanced Stability and Predominance of Period Doubling and Chaos with Flexible Exchange Rates
}

\author{
Toichiro Asada, ${ }^{1}$ Christos Douskos, ${ }^{2}$ and Panagiotis Markellos ${ }^{2}$ \\ ${ }^{1}$ Faculty of Economics, Chuo University, 742-1 Higashinakano, Hachioji, Tokyo 192-0393, Japan \\ ${ }^{2}$ Faculty of Engineering, University of Patras, 26500 Patras, Greece \\ Correspondence should be addressed to Panagiotis Markellos, p.markellos@des.upatras.gr \\ Received 16 May 2007; Accepted 17 March 2008 \\ Recommended by Masahiro Yabuta
}

We explore a discrete Kaldorian macrodynamic model of an open economy with flexible exchange rates, focusing on the effects of variation of the model parameters, the speed of adjustment of the goods market $\alpha$, and the degree of capital mobility $\beta$. We determine by a numerical grid search method the stability region in parameter space and find that flexible rates cause enhanced stability of equilibrium with respect to variations of the parameters. We identify the Hopf-Neimark bifurcation curve and the flip bifurcation curve, and find that the period doubling cascades which leads to chaos is the dominant behavior of the system outside the stability region, persisting to large values of $\beta$. Cyclical behavior of noticeable presence is detected for some extreme values of a state parameter. Bifurcation and Lyapunov exponent diagrams are computed illustrating the complex dynamics involved. Examples of attractors and trajectories are presented. The effect of the speed of adaptation of the expected rate is also briefly discussed. Finally, we explore a special model variation incorporating the "wealth effect" which is found to behave similarly to the basic model, contrary to the model of fixed exchange rates in which incorporation of this effect causes an entirely different behavior.

Copyright (c) 2008 Toichiro Asada et al. This is an open access article distributed under the Creative Commons Attribution License, which permits unrestricted use, distribution, and reproduction in any medium, provided the original work is properly cited.

\section{Introduction}

In this paper, we consider a three-dimensional discrete time model of an open economy with flexible exchange rates. The model is in fact the Kaldorian small open economy model with flexible exchange rates, expressed by Asada et al. [1] as a three-dimensional system of nonlinear difference equations. The corresponding discrete model of the system of fixed exchange rates was studied in Asada et al. [2,3], while a similar Kaldorian-type model in continuous time was studied in Asada [4]. The Kaldorian business cycle theory based on the original idea of Kaldor [5] has been developed recently by Lorenz [6], Gandolfo [7], Agliari 
and Dieci [8], among others. For a general discussion of analytical and numerical methods in the study of nonlinear dynamical systems in economics, we refer to Lorenz [9].

The present work is an extension of the work presented in Asada et al. [1]. We carry out an extensive numerical exploration of the model and consider the effects of the changes of parameter values on the stability of equilibrium of the system. The main model parameters considered are the speed of adjustment of the goods market $\alpha$ and the degree of capital mobility $\beta$. Using as our main tool a numerical grid-search method, we determine the stability region in the parameter space. We also identify analytically the Hopf bifurcation curve and the flip bifurcation curve, which form parts of the boundary of this region. We further compute bifurcation and Lyapunov exponent diagrams to obtain information on the asymptotic behavior of the system when equilibrium is no longer stable, that is, outside the stability region in the parameter space.

The model exhibits complex dynamics. Certain conclusions are drawn regarding the effects of parameter changes on the stability of equilibrium and on the predominant asymptotic behavior of the system of flexible exchange rates outside the stability region. We find that, by comparison to fixed exchange rates, flexible exchange rates cause increased stability of equilibrium with respect to variations of the model parameters. The predominant asymptotic behavior of the system of flexible exchange rates outside the stability region is found to be period doubling cascades leading to chaos. Examples of this behavior of the system are illustrated by means of bifurcation and Lyapunov exponent diagrams. For certain extreme values of a state parameter, cycles of noticeable size and persistence are found to occur and are similarly illustrated by means of bifurcation and Lyapunov exponent diagrams as well as diagrams of the cycles themselves in two-dimensional projections. The effect of the speed of adaptation of the expected rate on the stability of equilibrium is also briefly discussed. Finally, we explore briefly the special case (Model 2) incorporating the so-called wealth effect, which in the present case of flexible exchange rates is found to behave similarly to the basic model, contrary to the case of fixed exchange rates in which incorporation of the wealth effect causes an entirely different behavior of the system.

The paper proceeds as follows. In Section 2, we present the equations of the model and the specifications adopted. In Section 3, we determine the position and stability of equilibrium, and identify analytically the Hopf-Neimark bifurcation curve and the flip bifurcation curve, which constitute parts of the boundary of the stability region. The results of our exploration of the occurring asymptotic dynamical behavior of the model outside the stability region are presented, in the form of bifurcation and Lyapunov exponent diagrams, and are discussed in Section 4. In Section 5, we present some examples of chaotic attractors and some trajectories. In Section 6, we explore the occurrence of cycles; and in Section 7, we consider briefly the effect of the parameter $\gamma$, representing the speed of adaptation of the expected rate, on the stability of equilibrium. Finally, in Section 8, we explore briefly the special case (Model 2) incorporating the so-called wealth effect, and Section 9 concludes.

\section{Model equations-variables and parameters}

We consider the three-dimensional model of open economy Kaldorian dynamics proposed by Asada et al. [1]. This is obtained from the following system of equations:

$$
Y(t+1)-Y(t)=\alpha[C(t)+I(t)+G+J(t)-Y(t)], \quad \alpha>0,
$$


Toichiro Asada et al.

$$
\begin{gathered}
K(t+1)-K(t)=I(t), \\
C(t)=c[Y(t)-T(t)]+C_{0}, \quad 0<c<1, C_{0}>0, \\
I(t)=I[Y(t), K(t), r(t)], \quad \frac{\partial I}{\partial Y}>0, \frac{\partial I}{\partial K}<0, \frac{\partial I}{\partial r}<0, \\
T(t)=\tau Y(t)-T_{0}, \quad 0<\tau<1, T_{0}>0, \\
M(t)=p L[Y(t), r(t)], \quad \frac{\partial L}{\partial Y}>0, \frac{\partial L}{\partial r}<0, \\
J(t)=J[Y(t), E(t)], \quad \frac{\partial J}{\partial Y}<0, \frac{\partial J}{\partial E}>0, \\
Q(t)=\beta\left[r(t)-r_{f}-\frac{E^{e}(t)-E(t)}{E(t)}\right], \quad \beta>0, \\
A(t)=J(t)+Q(t), \\
A(t)=0, \\
E^{e}(t+1)-E^{e}(t)=r\left[E(t)-E^{e}(t)\right], \quad r>0, \\
M(t)=\bar{M},
\end{gathered}
$$

where $t$ denotes the time period and the meanings of the symbols are as follows. $Y=$ net real national income, $C=$ real consumption expenditure, $K=$ real physical capital stock, $T=$ real income tax, $M=$ nominal money supply, $I=$ net real private investment expenditure on physical capital, $G=$ real government expenditure (fixed), $p=$ price level (fixed), $E=$ exchange rate, $E^{e}=$ expected exchange rate in near future, $J=$ balance of current account (net export) in real terms, $Q=$ balance of capital account in real terms, $A=J+Q=$ total balance of payments in real terms, $r=$ nominal rate of interest, $r_{f}=$ nominal foreign rate of interest (fixed), $\alpha=$ adjustment speed of the goods market, and $\beta=$ degree of capital mobility. Finally, the parameter $\gamma$ represents the "speed of adaptation" of the expected rate.

Equation (2.1) expresses the Kaldorian adjustment process of the goods market, and (2.2) is the equation of capital accumulation, stating that the physical capital stock increases or decreases according to whether net investment is positive or negative. Equation (2.3) is the standard Keynesian consumption function, and (2.4) is the Kaldorian investment function. Equations (2.5) and (2.6) are the income tax function and the condition of equilibrium for the money market, respectively. Equation (2.7) is a standard type of the current account equation, stating that the current account is determined by $Y(t)$ and $E(t)$. Equation (2.8) expresses the idea that the capital account is positive or negative according to whether the difference between the rates of return of domestic and foreign exchange bonds is positive or negative. Equation (2.9) is the definition of the total balance of payments. Equation (2.10) characterizes the flexible exchange rate system, stating the assumption that the exchange rate is adjusted instantaneously to keep the equilibrium of the total balance of payments $[A(t)=0]$. Equation (2.11) expresses the "adaptive expectation hypothesis" concerning the expected exchange rate. Contrary to Asada et al. [1,2], in the present paper, we do not assume that the speed of adaptation $\gamma$ is fluctuated by noise. Finally, (2.12) expresses the institutional arrangements of the system of flexible exchange rates, stating that under flexible exchange rate the domestic monetary authority can control money supply contrary to the case of fixed exchange rate 
system, so that the money supply $M$ can be considered as an exogenous variable (to be assumed fixed: $M=\bar{M}$ ). The dynamical system can be described by the following equations:

$$
\begin{gathered}
Y(t+1)-Y(t)=\alpha\left[c(1-\tau) Y(t)+c T_{0}+C_{0}+G+\right. \\
I\{Y(t), K(t), r(Y(t), \bar{M})\}+J[Y(t), E(t)]-Y(t)], \\
K(t+1)-K(t)=I[Y(t), K(t), r(Y(t), \bar{M})], \\
E^{e}(t+1)-E^{e}(t)=r\left[E(t)-E^{e}(t)\right], \\
A(t)=J[Y(t), E(t)]+\beta\left\{r(Y(t), \bar{M})-r_{f}-\frac{E^{e}(t)}{E(t)}+1\right\}=0 .
\end{gathered}
$$

The first three of these equations are the difference equations describing the evolution of our discrete dynamical system, while the important quantity $E(t)$ is found at every "step" from (2.16). Further details on the present discrete model of flexible exchange rates and its similarities to a continuous time version are given in Asada et al. [1].

In the present paper, for our numerical exploration we adopt the same specifications of Asada et al. [1] as follows:

$$
\begin{gathered}
I[Y(t), K(t), r(t)]=f(Y(t))-0.3 K(t)-r(t)+100, \\
r(t)=r(Y(t), M)=10 \sqrt{Y(t)}-M, \\
J[Y(t), E(t)]=-0.3 Y(t)+100-\frac{100}{E(t)}, \\
c=0.8, \quad \tau=0.2, \quad p=1, \quad r_{f}=6, \quad M=\bar{M}=100, \quad r=1.2,
\end{gathered}
$$

except that we will consider the quantity $c T_{0}+C_{0}+G$ as a state parameter denoted by $Z$, and we will explore the following values for it:

$$
c T_{0}+C_{0}+G=Z=238,120,60,50 .
$$

The first value is the value proposed in Asada et al. [1]. Also, in Section 7, we will consider variation of the speed of adaptation $\gamma$ of the expected rate. We, thus, obtain the dynamical system described by the following nonlinear difference equations:

$$
\begin{gathered}
Y(t+1)-Y(t)=F_{1}=\alpha\left[-0.66 Y(t)+300+Z+f(Y(t))-0.3 K(t)-10 \sqrt{Y(t)}-\frac{100}{E(t)}\right] \\
K(t+1)-K(t)=F_{2}=f(Y(t))-0.3 K(t)-10 \sqrt{Y(t)}+200 \\
E^{e}(t+1)-E^{e}(t)=F_{3}=1.2\left[E(t)-E^{e}(t)\right]
\end{gathered}
$$

At every time step, the exchange rate is obtained from (2.16):

$$
E(t)=\frac{100+\beta E^{e}(t)}{100-105 \beta+10 \beta \sqrt{Y(t)}-0.3 Y(t)} .
$$


This system is called Model 1 to distinguish it from its variant, called Model 2, which we consider in Section 8. The function $f$ is a particular case of the Kaldorian S-shaped direct dependence of the investment function on income (see also Dohtani et al. [10], Agliari and Dieci [8], Asada et al. [3]) given by

$$
f(x)=\frac{80}{\pi} \arctan \left[\frac{9 \pi}{80}(x-112)\right]+35 .
$$

\section{Equilibrium position and stability}

It is easily found that the system is in equilibrium at

$$
\begin{gathered}
Y^{*}=\frac{25}{81}[9 Z+2 \beta\{477+625 \beta-25 \sqrt{9 Z+\beta(954+625 \beta)}\}], \\
K^{*}=\frac{10}{3}\left[200-10 \sqrt{Y^{*}}+f\left(Y^{*}\right)\right], \\
E^{e *}=E^{*}=\frac{1000}{1000-1060 \beta+100 \beta \sqrt{Y^{*}}-3 Y^{*}} .
\end{gathered}
$$

Indeed, (3.2) is obtained by solving $F_{2}=0$ for $K$, while (3.3) is similarly obtained by observing from (2.21) that the equilibrium values of $E^{e}$ and $E$ must be equal, and solving (2.22) for $E^{e}=E$. To find the equilibrium value of $Y$ we first solve $F_{2}=0$ for $f(Y)$, obtaining $f(Y)=$ $0.3 K+10 \sqrt{Y}-200$, and substitute this and (3.3) into $F_{1}=0$. We, then, obtain

$$
9 Y^{*}+250 \beta \sqrt{Y^{*}}-25(Z+106 \beta)=0,
$$

giving (3.1). Thus, for $Z=238, \beta=10$, we find that $Y^{*} \cong 154.36, K^{*} \cong 496.86$, and $E^{*} \cong 0.424$. All three equilibrium values depend on $\beta$, and they are shown graphically as functions of $\beta$ in Figure 1. We note that $Y^{*}$ and $K^{*}$ are always positive for $\beta>0$. However, $E^{*}$ is positive for $\beta>0$ only when $Z<120$. For $Z>120$, the value $E^{*}$ is positive only when $\beta>\beta_{\text {min }}>0$, where $\beta_{\text {min }}$ is the root of the denominator in (3.3):

$$
1000-1060 \beta+100 \beta \sqrt{Y^{*}}-3 Y^{*}=0 .
$$

For example, when $Z=238$, the minimum value of $\beta$ for which we have a positive equilibrium is $\beta_{\min } \cong 0.446$. More generally, it is easily found, by eliminating $Y^{*}$ from (3.4) and (3.5), that

$$
\beta_{\min }=\frac{15(Z-120)}{50 \sqrt{66} \sqrt{Z+100}-3498} .
$$

This is plotted in Figure 2.

Stability of the equilibrium is determined by the roots of the characteristic polynomial of the Jacobian of the mapping:

$$
\left(\begin{array}{ccc}
1+\frac{\partial F_{1}}{\partial Y} & \frac{\partial F_{1}}{\partial K} & \frac{\partial F_{1}}{\partial E^{e}} \\
\frac{\partial F_{2}}{\partial Y} & 1+\frac{\partial F_{2}}{\partial K} & \frac{\partial F_{2}}{\partial E^{e}} \\
\frac{\partial F_{3}}{\partial Y} & \frac{\partial F_{3}}{\partial K} & 1+\frac{\partial F_{3}}{\partial E^{e}}
\end{array}\right)
$$




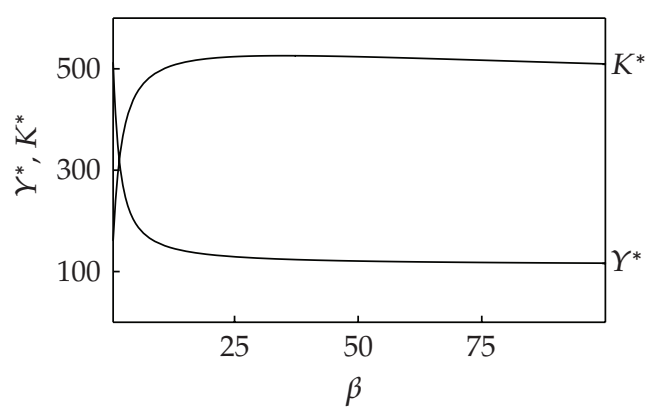

(a)

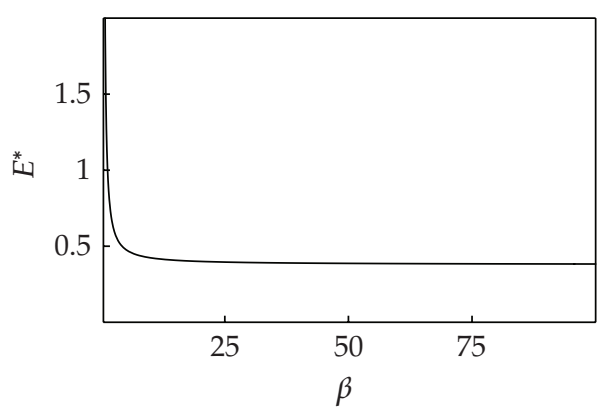

(b)

Figure 1: Equilibrium values as functions of $\beta$, for $Z=238$.

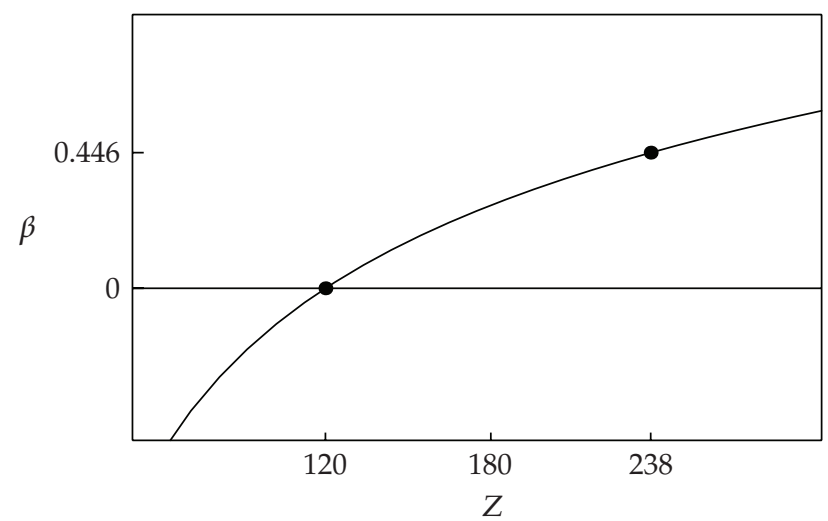

Figure 2: The minimum value of $\beta$ for positive equilibrium, as function of $Z$.

evaluated at the equilibrium. The characteristic equation is of the form

$$
P_{3}(\lambda)=\lambda^{3}+a_{2} \lambda^{2}+a_{1} \lambda+a_{0}=0,
$$

and the Cohn-Shur conditions for stability are

$$
1+a_{1}-\left|a_{2}+a_{0}\right|>0, \quad 1-a_{1}+a_{2} a_{0}-a_{0}^{2}>0, \quad a_{1}<3 .
$$

These conditions represent relations between the parameters $\beta$ and $\alpha$ of the model which are satisfied in the shaded regions of the $(\beta, \alpha)$ plane shown in Figures 3 and 4 for the first two values of the parameter $Z$ explored here.

Our basic tool for the numerical determination of the region of stability is a gridsearch method. This involves computing the characteristic polynomial (3.8) and its roots at all node points of a dense grid covering a region in a two-dimensional space of parameters, and storing the points at which the equilibrium is stable. The technique is employed to determine the stability region diagrams of Figures 3 and 4 . The part of the stability region in which the roots of the characteristic equation are all real is shown dark shaded, while the part in which two of the roots are complex conjugate is shown light shaded.

The flip bifurcation curve,

$$
P_{3}(-1)=1+a_{1}-\left(a_{2}+a_{0}\right)=0,
$$




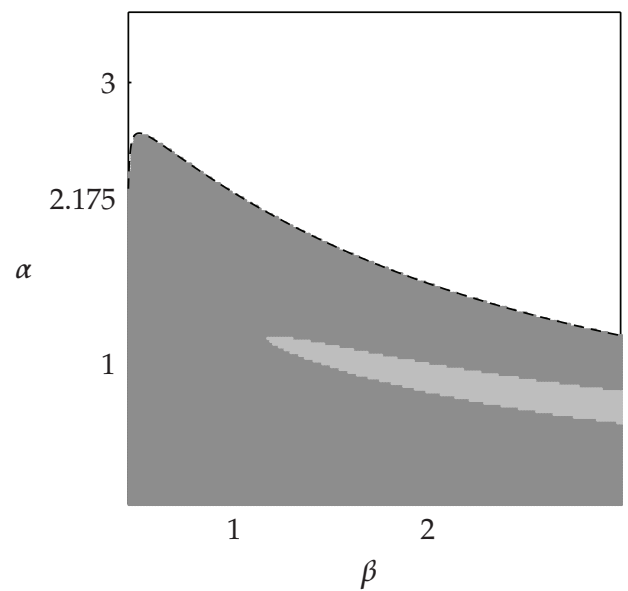

(a)

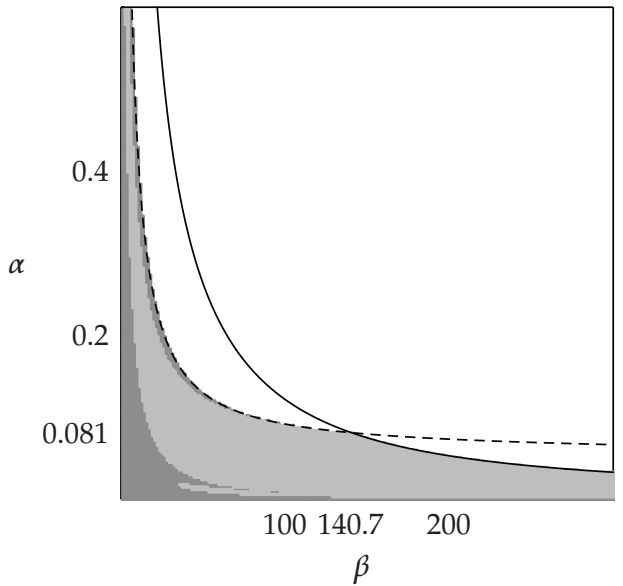

(b)

Figure 3: Region of stability of the equilibrium and its bounding curves, the flip bifurcation curve (dashed), and the Hopf-Neimark bifurcation curve (continuous), in the $(\beta, \alpha)$ parameter plane for $Z=238$.

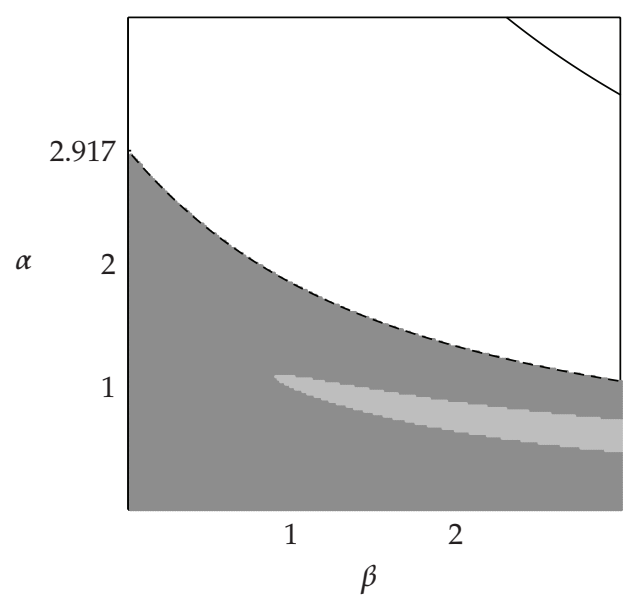

(a)

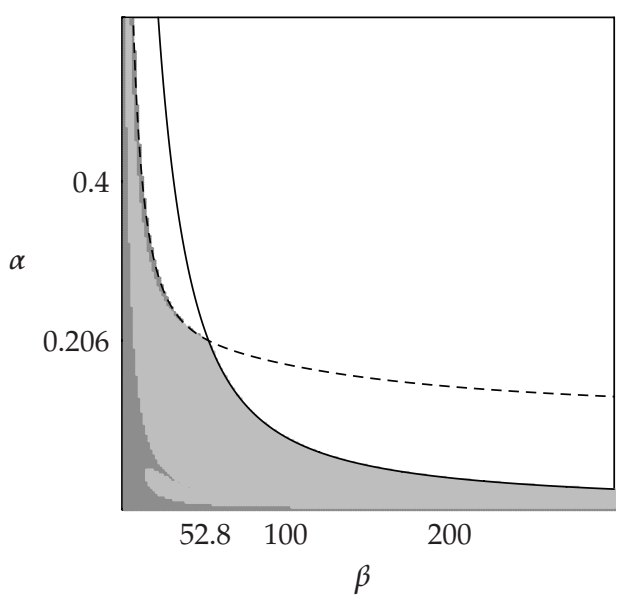

(b)

Figure 4: Region of stability of the equilibrium and its bounding curves, in the $(\beta, \alpha)$ parameter plane for $Z=120$.

related to the first Cohn-Shur condition for stability (3.9), is drawn in as a dashed curve, and the Hopf bifurcation curve is drawn in as a continuous curve. We have found that the Hopf bifurcation curve can be determined in the present case by requiring simply that two of the roots of the characteristic polynomial have unit product:

$$
\lambda^{3}+a_{2} \lambda^{2}+a_{1} \lambda+a_{0}=(\lambda-\rho)\left(\lambda^{2}+\mu \lambda+1\right) .
$$

The coefficients of the left-hand side are functions of the model parameters $\alpha$ and $\beta$. We, then, equate coefficients of same powers of $\lambda$, eliminate $\rho$ and $\mu$, and obtain the condition:

$$
1-a_{1}+a_{2} a_{0}-a_{0}^{2}=0 .
$$


This is the implicit equation of the Hopf bifurcation curve in the present stability diagrams, and we note that it is related to the second Cohn-Shur condition (3.9).

The intersection of the two curves, forming parts of the boundary of the stability region, occurs at the point indicated, in the diagrams of Figures 3 and 4 , by its coordinate values: $(140.7,0.081)$ for $Z=238$, and $(52.8,0.206)$ for $Z=120$. This shows that up to such large values of the degree of capital mobility $\beta$ stability of equilibrium is lost through a flip bifurcation. Therefore, up to such large values of the degree of capital mobility $\beta$ period doubling can be expected to occur outside the stability region.

In Asada et al. [1], the conclusion was drawn by analytical considerations regarding the stability of the system in the $(\beta, \alpha)$ plane, that under certain conditions the increase of the adjustment speed of the goods market $\alpha$ and the degree of capital mobility $\beta$ tends to destabilize the system of flexible exchange rates, in the sense that the equilibrium point becomes unstable for sufficiently large $\alpha$ and $\beta$. In the present paper, we see that in fact, for the specifications adopted in the present formulation of the model, increase of the parameter $\alpha$ destabilizes the equilibrium quickly for large values of $\beta$, while high levels of the adjustment speed $\alpha$ are required for destabilization for relatively low levels of capital movement $\beta$. On the other hand, increase of the parameter $\beta$, to very high values, does not destabilize the equilibrium for sufficiently small values of $\alpha$. We recall that a small value of $\alpha(<1)$ implies prudent reaction by firms, while a large value $(\alpha>1)$ implies rash reaction and coordination failure (see Agliari and Dieci [8]). Thus, one might interpret the stability region by saying that capital movement, even at very high levels, does not destabilize the system of flexible exchange rates so long as firms are sufficiently prudent in their reactions.

These results are contrary to the corresponding behavior of the fixed exchange rates system, in which the equilibrium is destabilized at much lower levels of capital movement and/or adjustment speed (see Asada et al. [3]). Thus, flexibility of the exchange rates causes enhanced stability of equilibrium with respect to variations of the basic parameters of the model.

\section{Bifurcation and Liapunov exponent diagrams}

To illustrate the complicated dynamics involved, we have employed numerical simulations of the trajectories to obtain characteristic bifurcation and Lyapunov exponent diagrams representing the asymptotic state of the system. In Figures 5 and 6, we present such diagrams for $Z=238$ and some sample values of the parameter $\beta$, with the parameter $\alpha$ as the bifurcation parameter. Similarly, in Figure 7, we present such diagrams for $Z=120$ and sample values of the parameter $\beta$. Some details of the period-doubling process for $Z=238$ and $\beta=20$ are shown in Figure 8 .

These diagrams show that, as expected from the region of stability diagrams, the predominant behavior of the system of flexible exchange rates for parameter values outside the stability region is that of period-doubling cascades leading to chaos. The existing windows of periodicity are also visible within the chaotic regions. The Lyapunov exponent diagrams clearly indicate chaotic behavior when it occurs. We also note in Figures 5 and 6 that for $Z=238$ period-doubling behavior persists longer (for larger intervals of $\alpha$ ) at higher levels of capital mobility.

\section{Examples of attractors and trajectories}

In Figure 9, we present two examples of chaotic attractors of the system of flexible exchange rates, in the case of $Z=120$ and $\beta=5$. The first example corresponds to a two-piece attractor, 
Toichiro Asada et al.

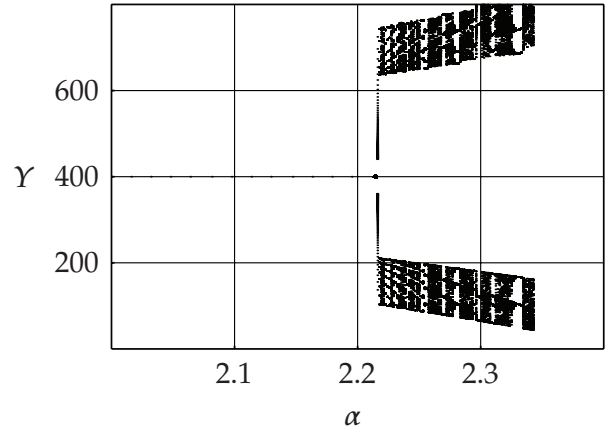

(a)

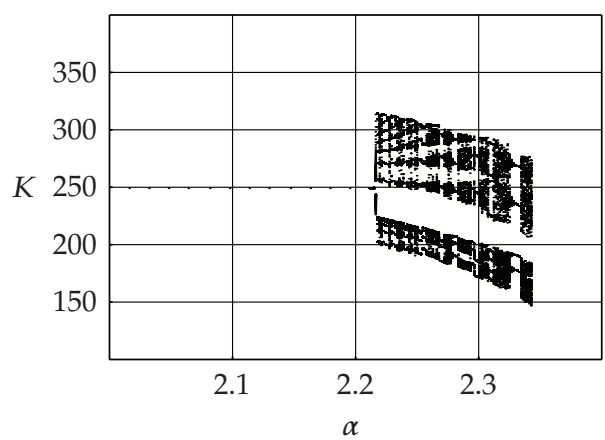

(c)

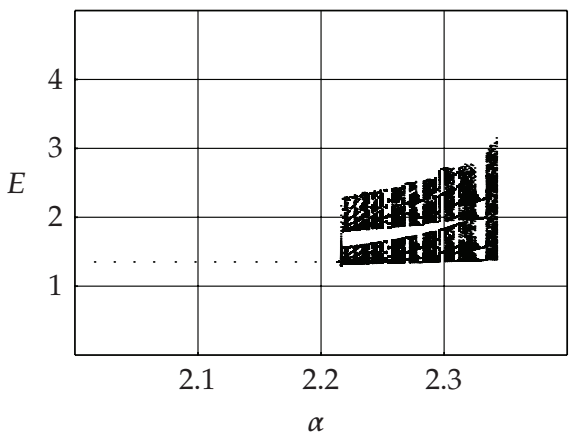

(e)

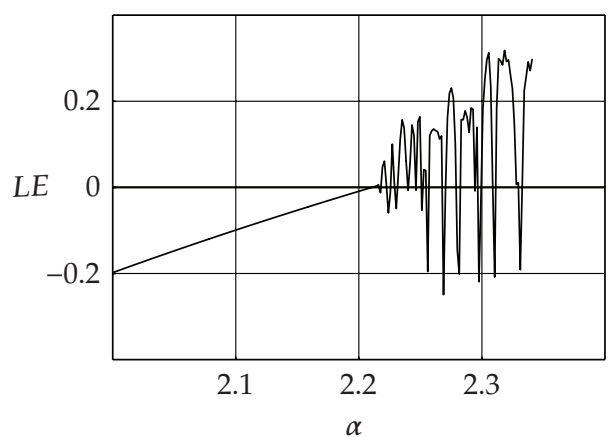

(g)

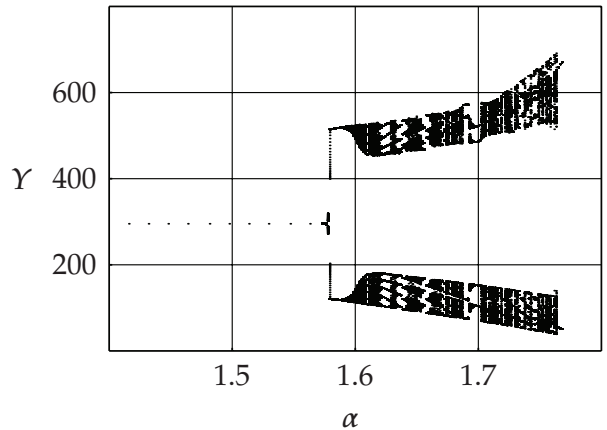

(b)

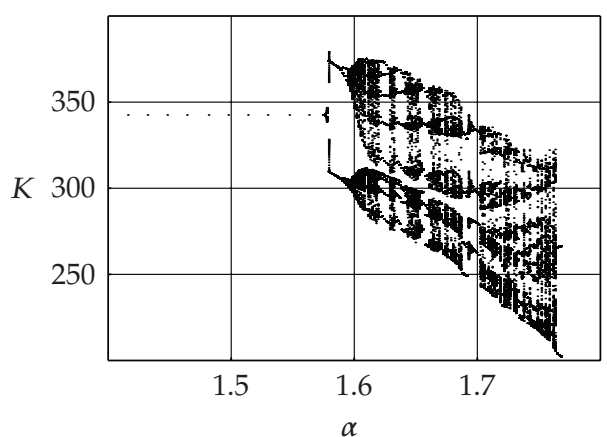

(d)

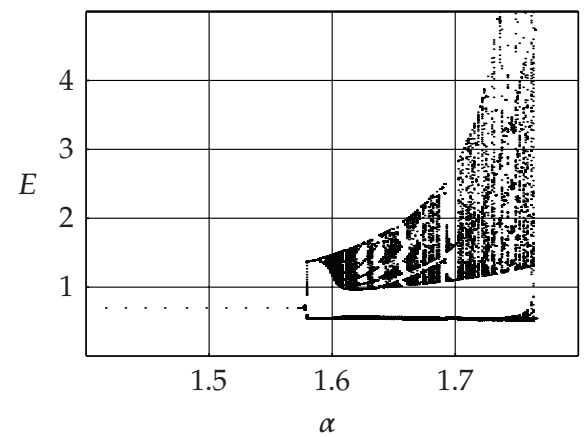

(f)

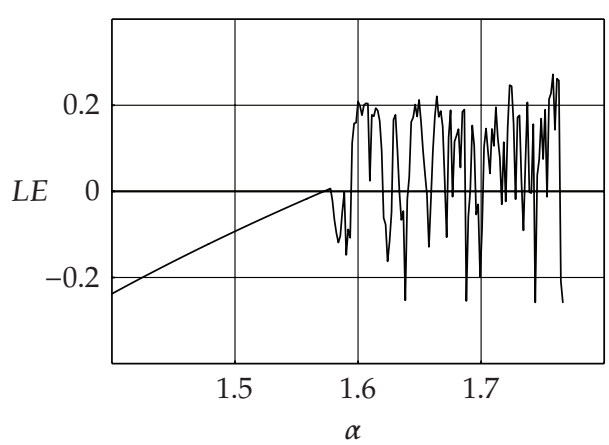

(h)

Figure 5: Bifurcation diagrams and Lyapunov exponent for $Z=238$ : $\beta=1$ (left) and $\beta=2$ (right). 


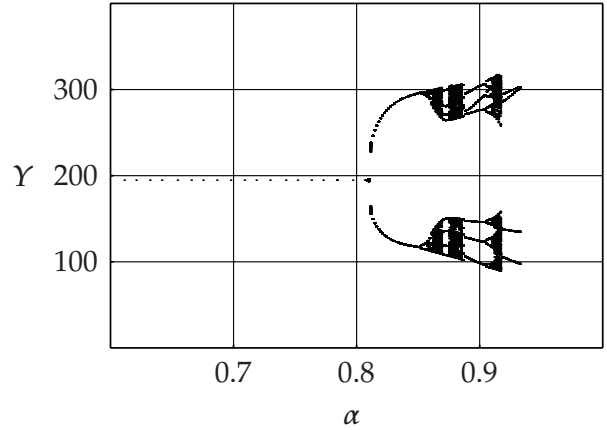

(a)

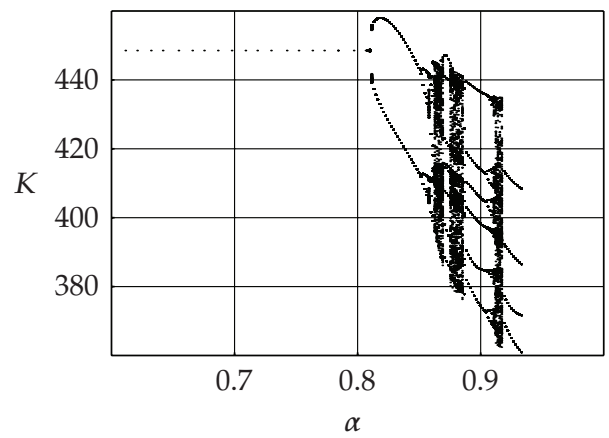

(c)

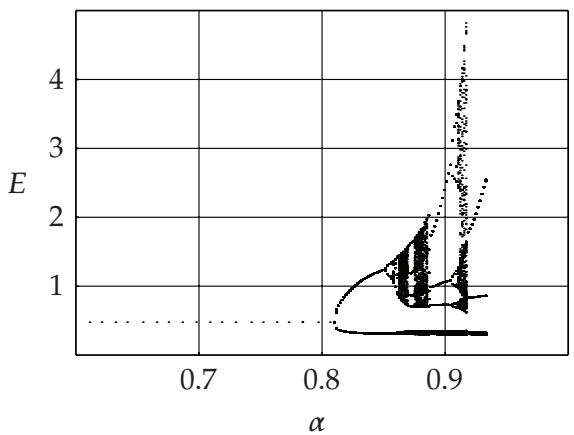

(e)

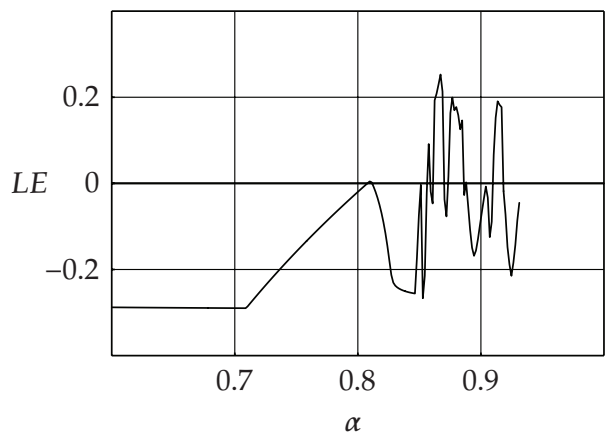

(g)

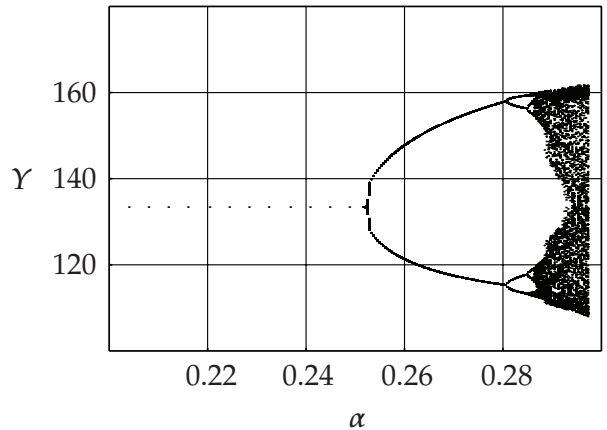

(b)

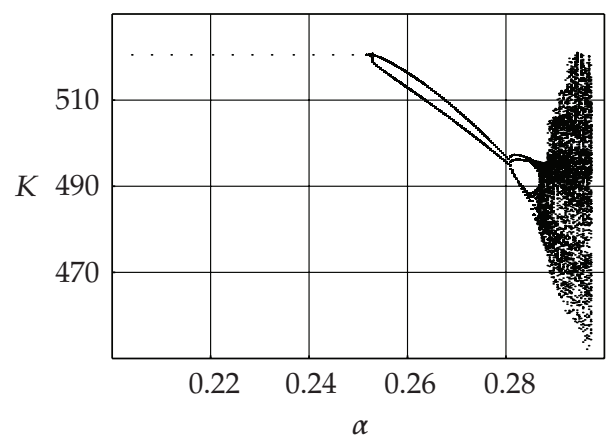

(d)

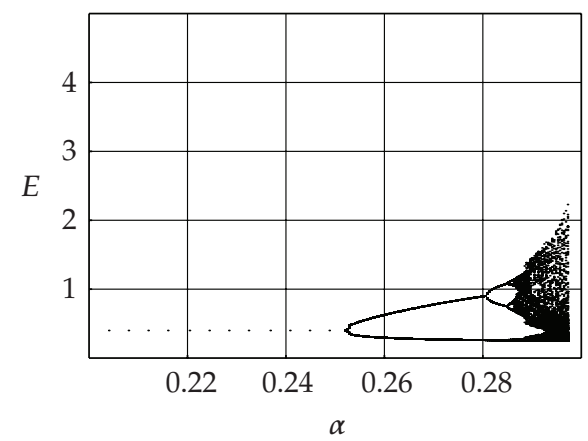

(f)

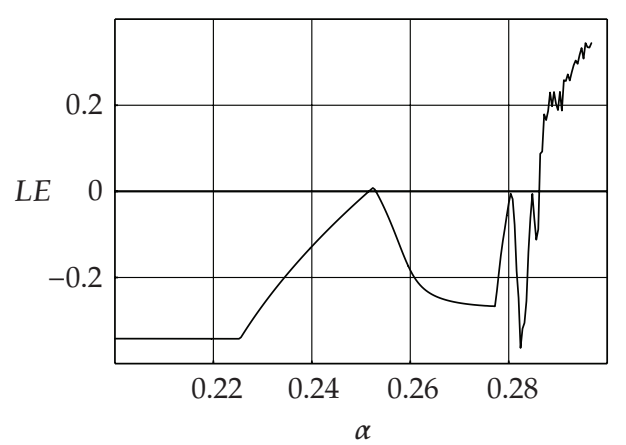

(h)

Figure 6: Bifurcation diagrams and Lyapunov exponent for $Z=238$ : $\beta=5$ (left) and $\beta=20$ (right). 


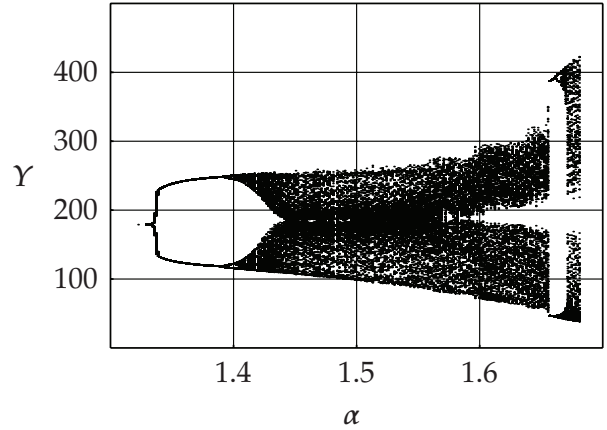

(a)

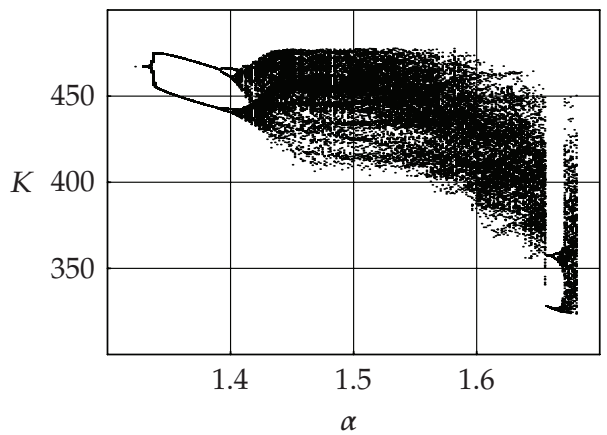

(c)

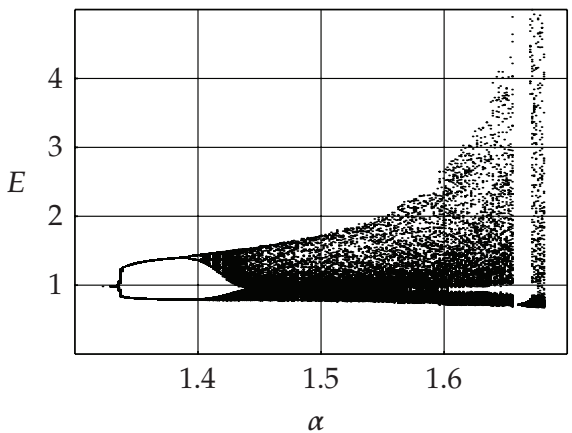

(e)

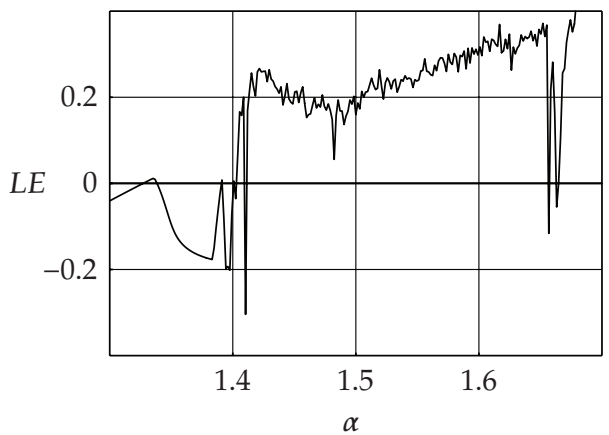

(g)

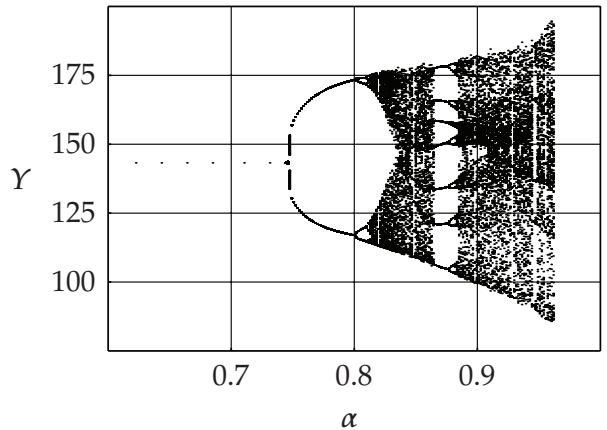

(b)

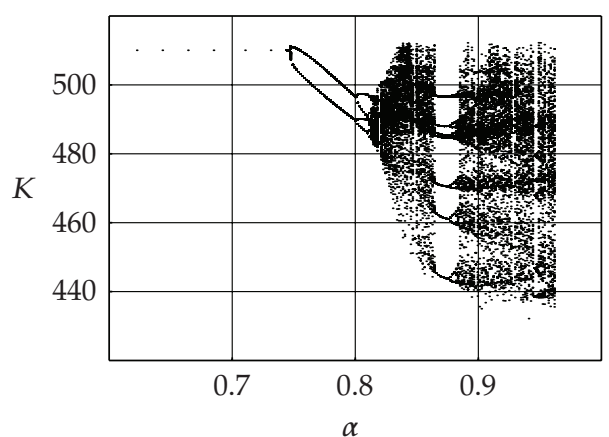

(d)

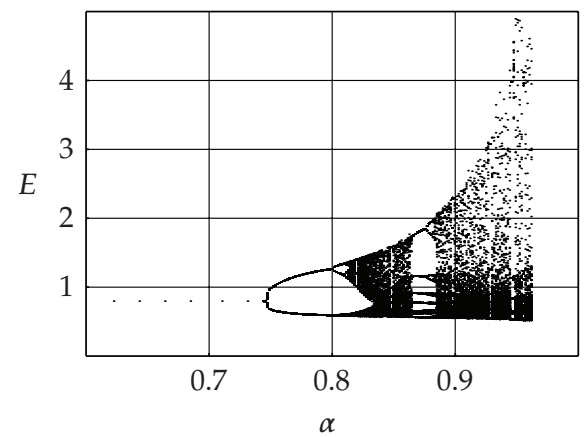

(f)

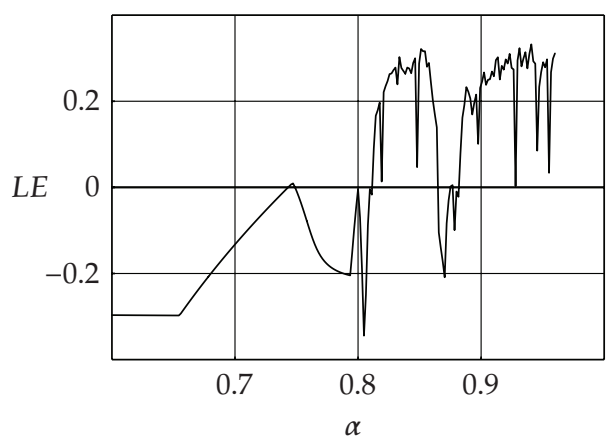

(h)

Figure 7: Bifurcation diagrams and Lyapunov exponent for $Z=120: \beta=2$ (left) and $\beta=5$ (right). 


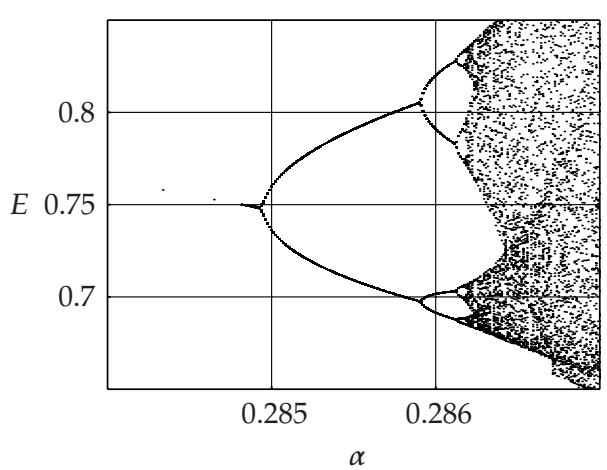

(a)

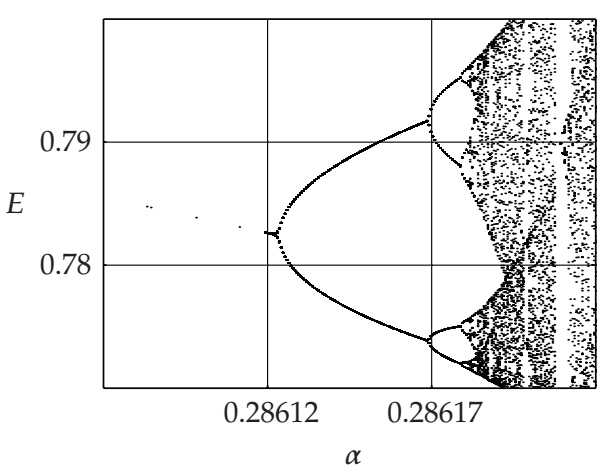

(b)

Figure 8: Details of the bifurcation diagram (Figure 6) of $E$ for $Z=238, \beta=20$ (two levels of magnification).

while the second example shows the evolution of that attractor when its two pieces have merged into a single chaotic attractor.

The two dominant types of dynamical behavior exhibited by the system are shown in Figure 10 in terms of the actual time paths-trajectories-of the capital stock and the exchange rate. In the top row, we see the case of a periodic trajectory (the trajectory of a 7-periodic orbit), while, in the bottom row, we see the case of a chaotic trajectory.

\section{The occurrence of cycles}

In Figures 3 and 4 , for $\beta>140.7$ and $\beta>52.8$, respectively, cycles would be expected to occur when crossing the Hopf bifurcation curve, but such cycles were found to be difficult to compute, being small in size and existing very briefly, that is, for very small interval of values of the parameter $\alpha$ after crossing the bifurcation curve. Nevertheless, we have found cycles of noticeable size, and existing for intervals of $\alpha$ values of noticeable length, at smaller values of the state parameter $c T_{0}+C_{0}+G=Z$. For this, we have used the stability region diagrams for $Z=50$ and $Z=60$ (Figure 11), which we determined by the same grid-search method as before. In these cases, the flip bifurcation curve forms part of the stability region boundary only at relatively low values of $\beta$.

Note that the region of stability diagrams for $Z=238,120$, and 60 are qualitatively similar as determined by the behavior of the bounding curves, but at $Z=50$ we meet with a qualitatively different behavior of the bounding curves. Also, it is at $Z=50$, and to a lesser extent at $Z=60$, among the four values considered for $Z$, that cycles of noticeable size and existing for intervals of $\alpha$ values of noticeable length were found. This can be seen, for example, in the bifurcation diagrams of Figures 12 and 13. Note also the characteristic flatness at the zero value of the Liapunov exponent diagrams in Figure 13, a landmark of cyclical behavior. The cycles themselves for $Z=50$ are shown for two different values of $\beta$ in Figures 14 and 15. A chaotic attractor showing the chaotic asymptotic behavior of the system after the development of the cycles, for $Z=50, \beta=5, \alpha=0.8$, is also shown in Figure 15.

As a matter of interest, we note that, in the case $Z=50$ and $\beta=5$, the bifurcation appears to be of the subcritical type. This type of bifurcation is of particular interest to economists as well as to mathematicians, as it may be associated to a double bifurcation process, creating coexistence of attractors, related hysteresis effects and policy questions 


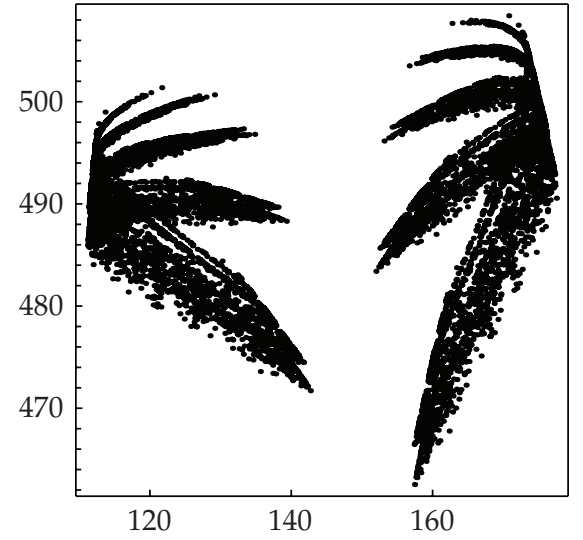

(a)

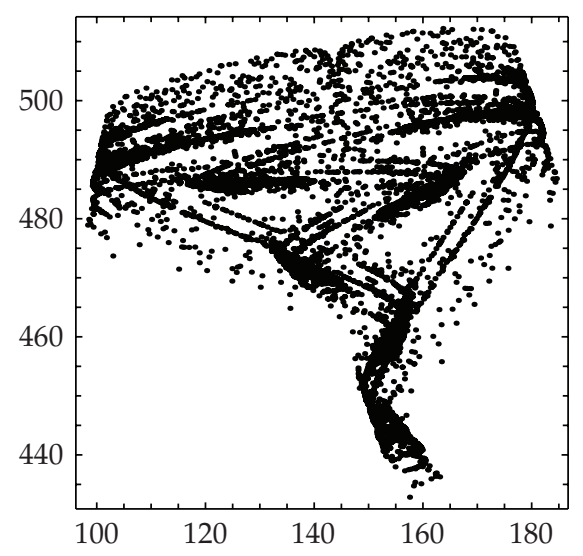

(c)

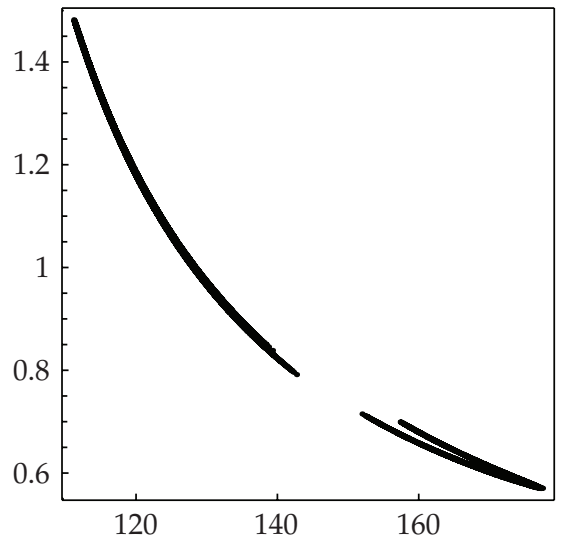

(b)

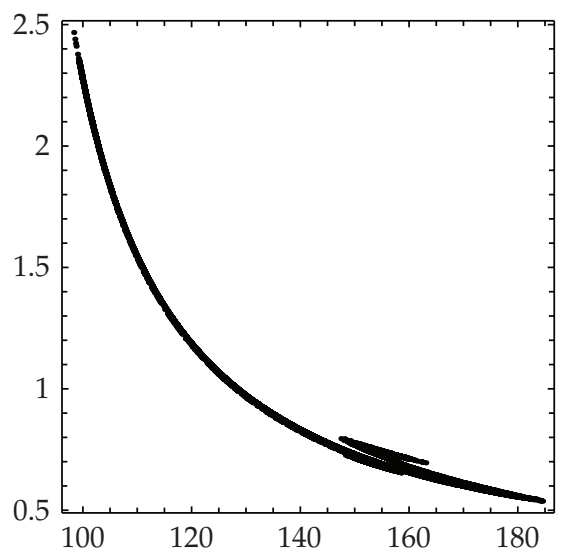

(d)

Figure 9: Chaotic attractors in their projections in the $(Y, K)$ plane (left) and the $(Y, E)$ plane (right), for $Z=120, \beta=5$, and $\alpha=0.83$ (top), $\alpha=0.9$ (bottom).

(see, e.g., Kind [11], Agliari [12]). The occurrence of such a bifurcation in the present case is supported by the numerical evidence of Figure 16.

A description of this evidence is as follows. The right diagram shows three attractors found by starting the iteration process very near the equilibrium point, for three values of the bifurcation parameter near the value $\alpha=0.3647$ at which stability is lost for the present values of the other parameters. The first value $\alpha=0.3645$ corresponds to stable equilibrium and the iterations end at a single point, the equilibrium point. The next two values, $\alpha=0.3650$ and 0.3655 , correspond to unstable equilibrium and the iterations accumulate on cycles, but these cycles appear by their sizes to represent a discontinuous jump from the equilibrium point, and this is made apparent by the fact that they are also very close to each other in spite of the fact that the change in parameter value between them is the same as the change of parameter value between the first cycle and the equilibrium point. This apparent discontinuity suggests that the bifurcation is subcritical. The evidence is more conclusive in the left diagram of Figure 16, produced by starting the iterations far from the equilibrium. In this diagram, we see that the same situation occurs at three values of the bifurcation 


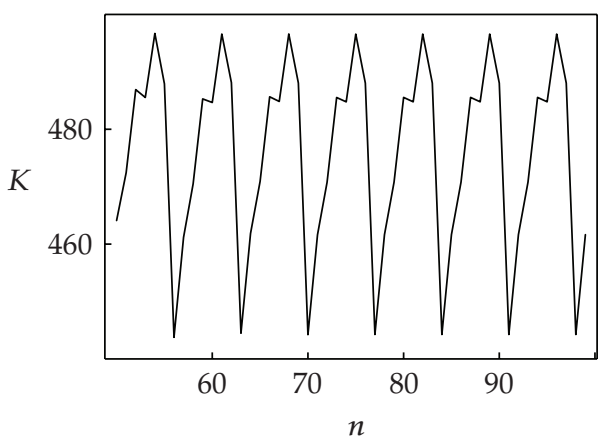

(a)

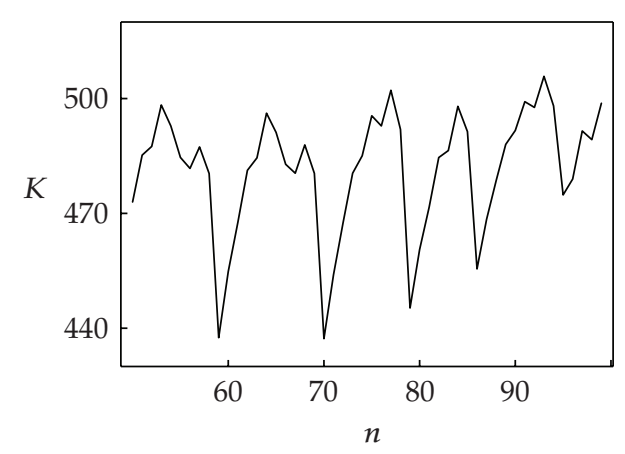

(c)

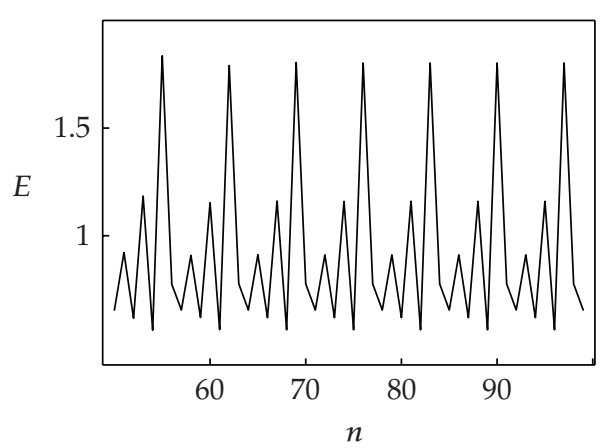

(b)

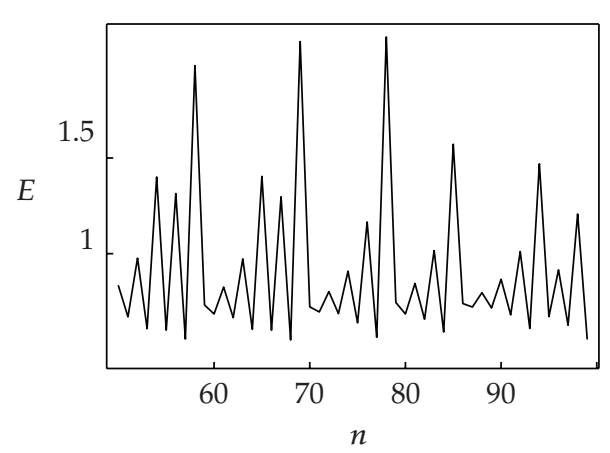

(d)

Figure 10: Trajectories of $K$ and $E$ for $Z=120, \beta=5$. Top: regular (7-periodic), $\alpha=0.87$. Bottom: chaotic, $\alpha=0.9$.

parameter all of which correspond to stable equilibrium. The cycles have appeared inside the stability region. Thus, the evidence of Figure 16 points to a double bifurcation process in which a global bifurcation occurs inside the stability region and a local bifurcation occurs when equilibrium loses stability. During this process, we have coexistence of a point attractor and a cyclical attractor for an interval of values of the bifurcation parameter. For further discussion on the subcritical Hopf bifurcation, see Puu [13].

\section{Effect of the speed of adaptation of the expected rate}

We now consider briefly the effect of the parameter $\gamma$, representing the speed of adaptation of the expected rate, on the stability of equilibrium. To do this, we use the same gridsearch method, together with the analytic determination of the flip bifurcation curve and the Hopf bifurcation curve, to determine the region of stability in the $(\beta, \gamma)$ plane for $Z=238$ and several values of the parameter $\alpha$. Some of the stability regions obtained are shown in Figure 17, while qualitatively similar results are obtained for other values of the state parameter $Z$.

We recall here that in Asada et al. [1] the conclusion was drawn by analytical considerations regarding the stability of the system in the $(\beta, \gamma)$ plane, that under certain conditions the equilibrium is locally unstable when the parameters $\beta$ and $\gamma$ are sufficiently large. In the present paper, we see that in fact, for the specifications adopted in the present formulation of the model, this conclusion is confirmed although one of the sufficient 


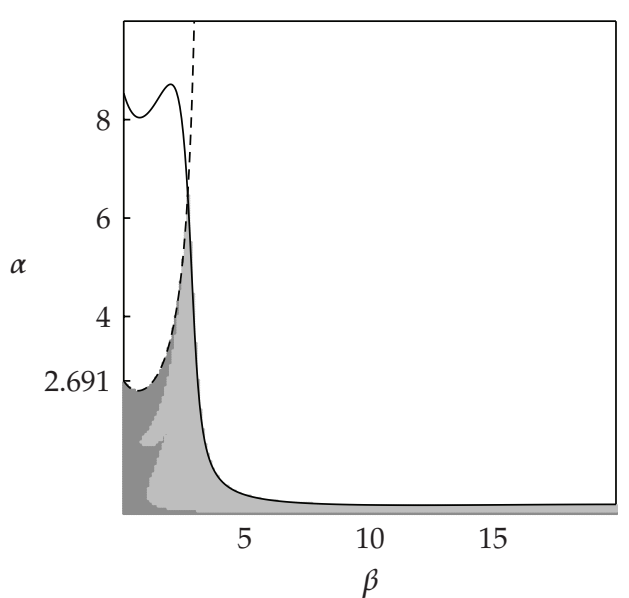

(a)

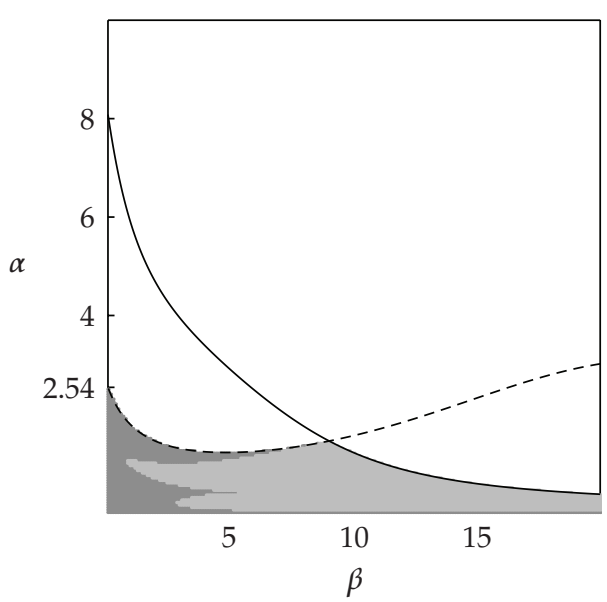

(b)

Figure 11: Region of stability of the equilibrium and its bounding curves, the flip bifurcation curve (dashed) and the Hopf-Neimark bifurcation curve (continuous), in the $(\beta, \alpha)$ parameter plane for $Z=50$ (left) and $Z=60$ (right).

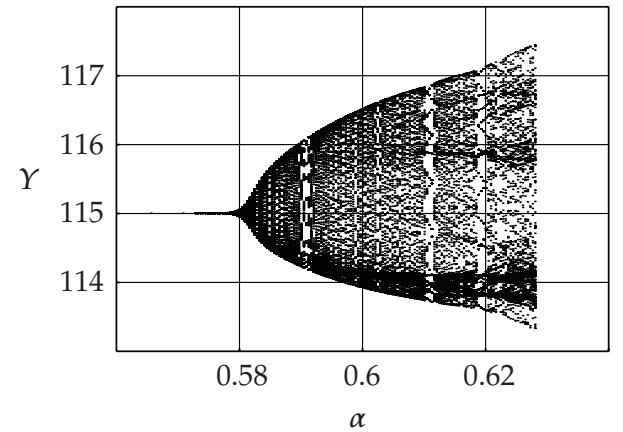

(a)

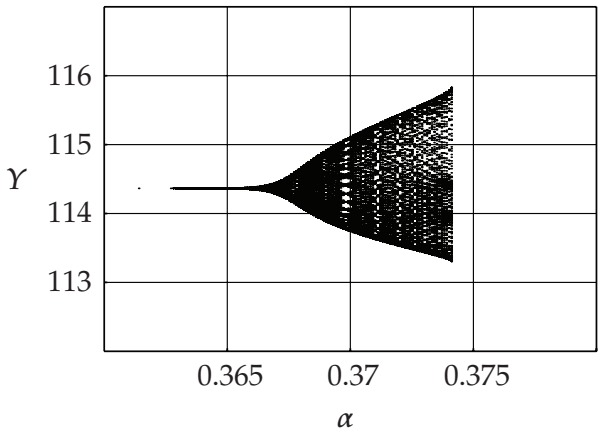

(b)

Figure 12: Bifurcation diagrams of $Y$ for $Z=60: \beta=15$ (left) and $\beta=20$ (right).

conditions $(\partial I / \partial K<-1)$ specified in Asada et al. [1] does not hold. Further, we see that for most levels of the speed of adjustment $\alpha \geq 0.5$ stability of equilibrium persists for values of the speed of adaptation $\gamma$ of the expected rate such that $\gamma<2$ approximately.

We also note that after a certain value of the adjustment speed $\alpha$, the entire stability region boundary is formed by the flip bifurcation curve (3.10), which now has the form

$$
P_{3}(-1)=\psi_{0}(\alpha, \beta)+\psi_{1}(\alpha, \beta) \gamma=0 .
$$

This relation can be written as

$$
r=-\frac{\psi_{0}(\alpha, \beta)}{\psi_{1}(\alpha, \beta)}=\frac{\zeta_{0}(\beta)+\zeta_{1}(\beta) \alpha}{\chi_{0}(\beta)+\chi_{1}(\beta) \alpha}
$$

and represents the two branches of a hyperbola-like curve (dashed) in each frame of Figure 17. 


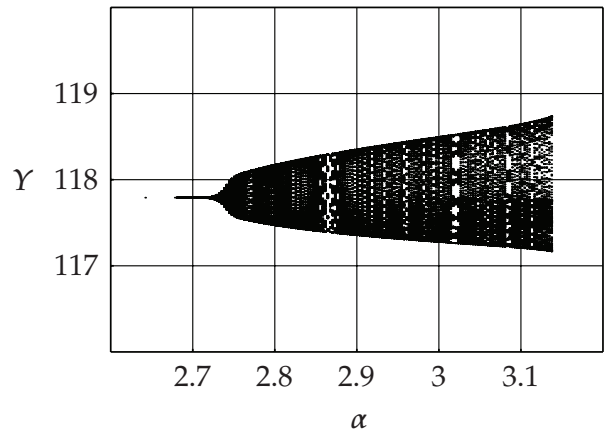

(a)

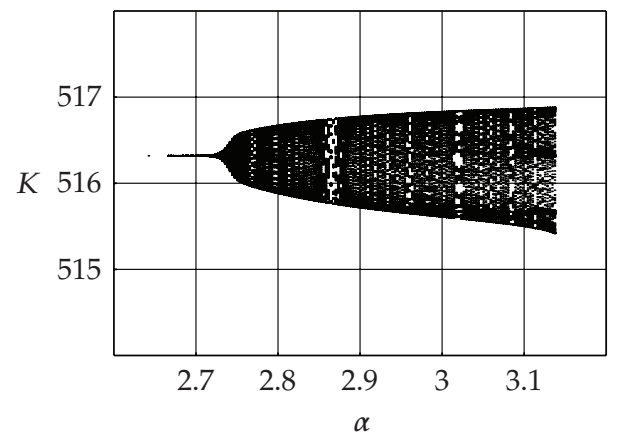

(c)

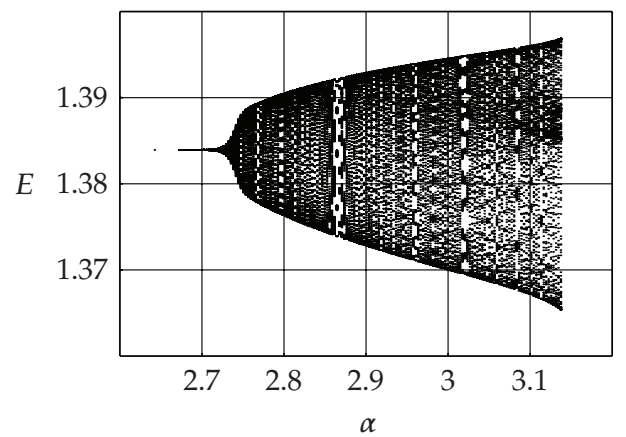

(e)

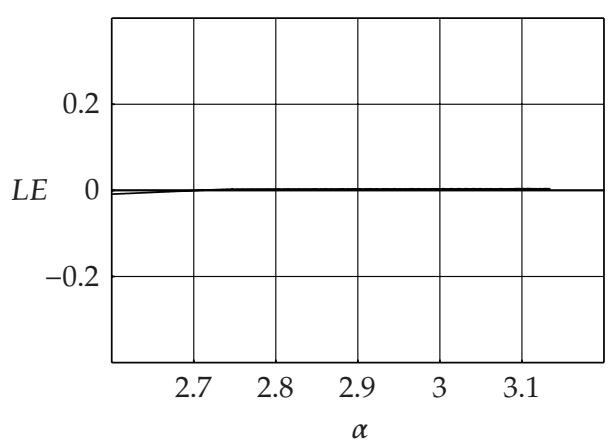

(g)

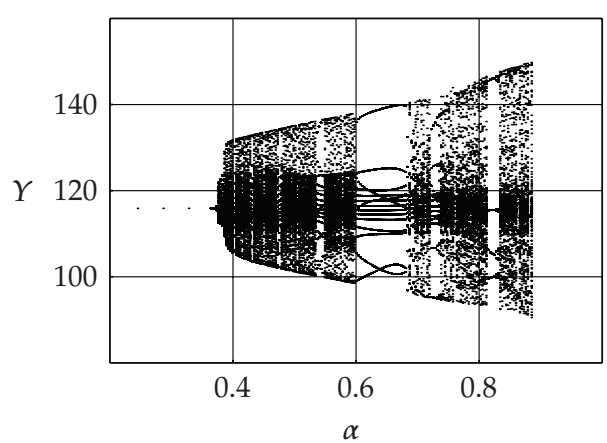

(b)

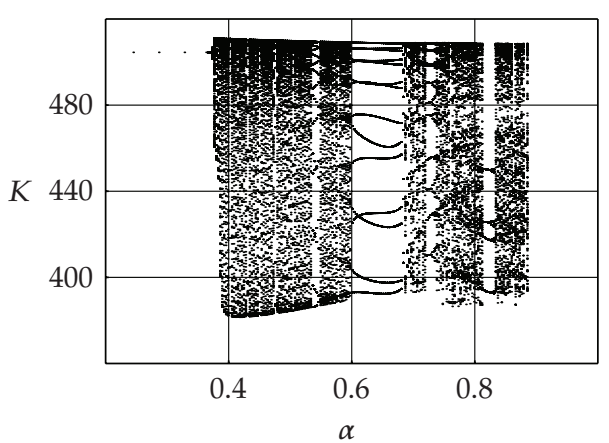

(d)

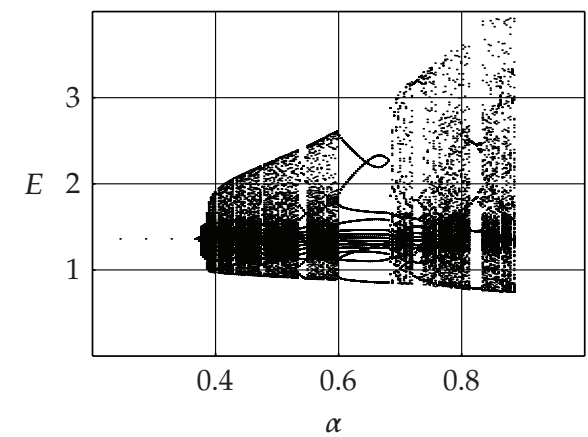

(f)

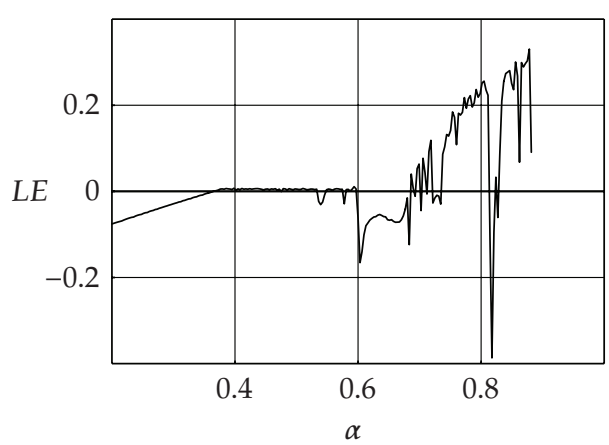

(h)

Figure 13: Bifurcation diagrams and Lyapunov exponent for $Z=50$ : $\beta=3$ (left) and $\beta=5$ (right). 
Toichiro Asada et al.

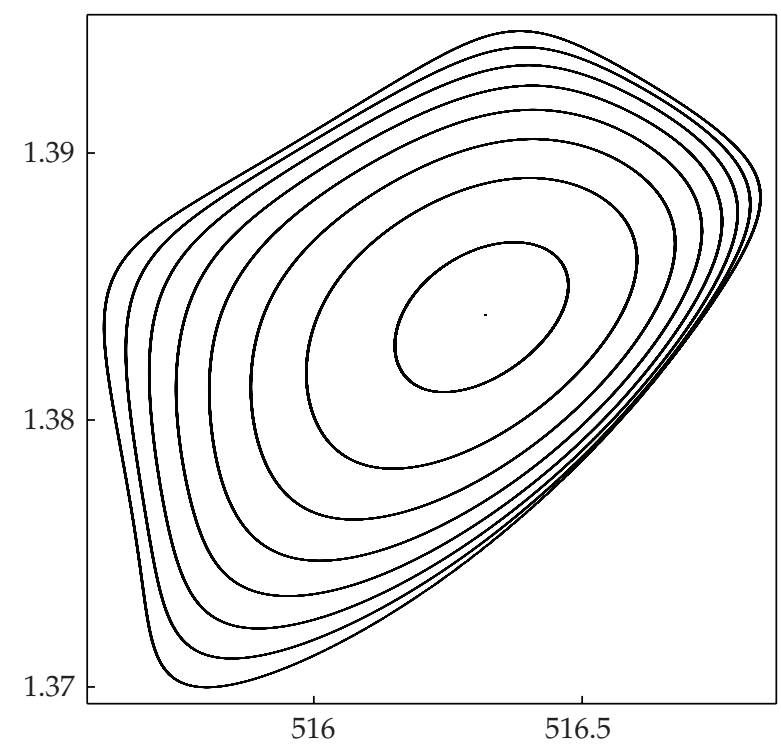

Figure 14: Development of cycles for $Z=50, \beta=3,2.7 \leq \alpha \leq 3$. Projection in the $(K, E)$ plane.

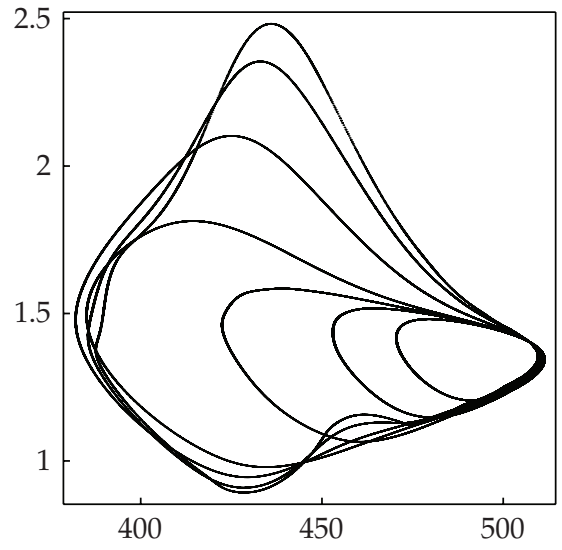

(a)

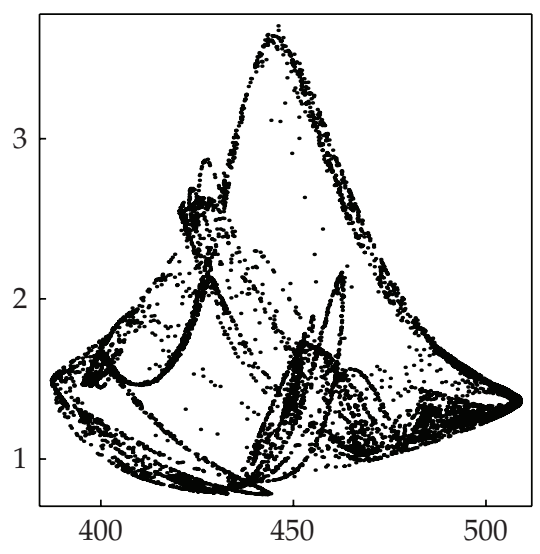

(b)

Figure 15: Left: development of cycles for $Z=50, \beta=5,0.37 \leq \alpha \leq 0.56$. Right: chaotic attractor for $Z=50, \beta=5, \alpha=0.8$. Projection in the $(K, E)$ plane.

Due to the linearity of numerator and denominator of the right-hand side with respect to $\alpha,(7.2)$ represents a hyperbola in the $(\alpha, \gamma)$ plane. We see that the topology of the curves in the $(\beta, \gamma)$ parameter plane changes at a critical value of $\alpha$, between $\alpha=1.5$ and $\alpha=2.2$. This happens when the two branches of the hyperbola in the $(\alpha, \gamma)$ plane meet, and the condition for the branches to meet is

$$
\left|\begin{array}{ll}
\zeta_{0}(\beta) & \zeta_{1}(\beta) \\
\chi_{0}(\beta) & \chi_{1}(\beta)
\end{array}\right|=0 .
$$




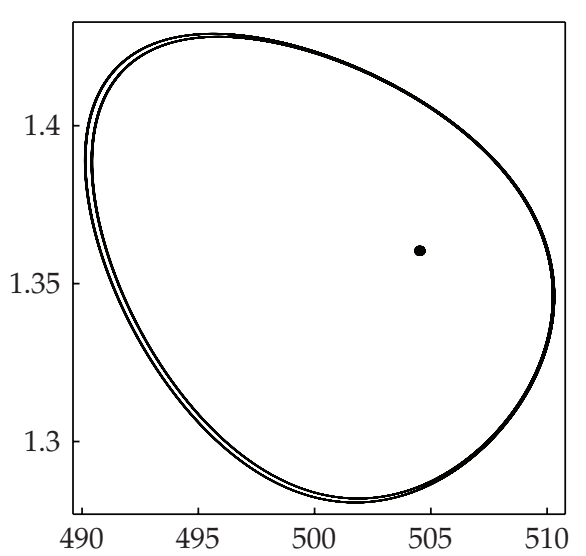

(a)

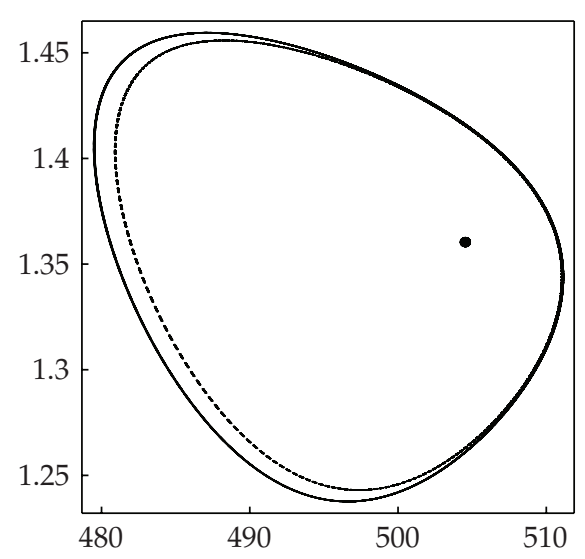

(b)

Figure 16: Graphical evidence of subcriticality of the Hopf bifurcation for $Z=50, \beta=5$. Left: three successive attractors (the first being a point) for $\alpha=0.363380,0.363385,0.363390$. Right: the same for $\alpha=0.3645,0.3650,0.3655$. Projections in the $(K, E)$ plane.

Solving this numerically we obtain the critical value of $\beta$ :

$$
\beta_{0} \cong 1.164,
$$

and the corresponding values of $\alpha$ and $\gamma$ are

$$
\alpha_{0}=-\frac{\zeta_{0}\left(\beta_{0}\right)}{\zeta_{1}\left(\beta_{0}\right)} \cong 2.079, \quad \gamma_{0}=\frac{\zeta_{1}\left(\beta_{0}\right)}{\chi_{1}\left(\beta_{0}\right)} \cong 2.026 \text {. }
$$

In the present case $(Z=238)$ and for values of $\alpha$ sufficiently larger than $\alpha_{0}$, specifically for $\alpha>2.0851$, the Hopf bifurcation curve no longer crosses the flip bifurcation curve and the entire boundary of the stability region is formed by the latter.

\section{Model 2-the "wealth effect"}

We now discuss, in the present case of flexible exchange rates, the special two-dimensional variation of the model, called Model 2 (Asada et al. $[2,3]$ ). The system of equations for model 2 is identical to $(2.19)-(2.22)$, except that the term $-0.3 K(t)$ is missing from (2.19). This means that the system decouples with (2.19) and (2.21) becoming independent of K. For economic theoretical justification of Model 2 see Asada et al. [2], where relevance of this model is discussed in relation to a special case of a type of savings function considered by Chang and Smyth [14], leading to a consumption function representing the "wealth effect" (according to which increase of the real capital stock stimulates the consumption expenditure).

Although Model 2 is of lower dimension than Model 1, consisting essentially of (2.19) and (2.21), determination of the equilibrium values as functions of the parameters is more difficult. However, we can still compute the equilibrium value of $E$ from (3.3) when the equilibrium value of $Y$ is known, and substituting (3.3) into $F_{1}^{(2)}=0$, where $F_{1}^{(2)}$ is $F_{1}$ for Model 2 (i.e., without the term $-0.3 K(t)$ ); we obtain the following equation that can be solved numerically for the equilibrium value $Y^{*}$ of $Y$ :

$$
200+106 \beta+Z-10(1+\beta) \sqrt{Y^{*}}-0.36 Y^{*}+f\left(Y^{*}\right)=0 .
$$


Toichiro Asada et al.

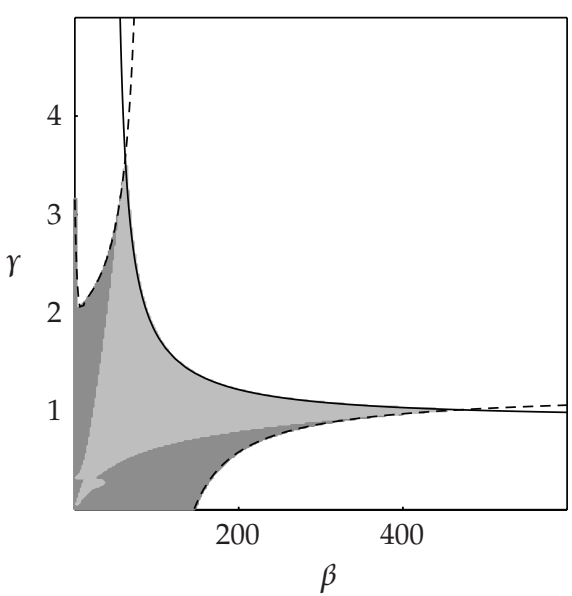

(a)

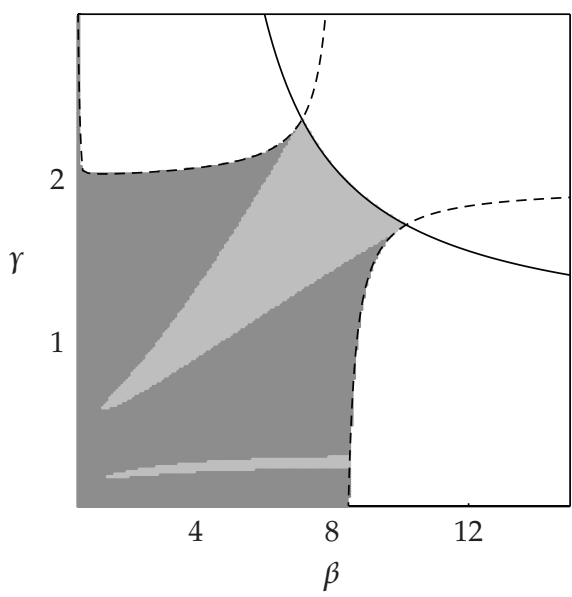

(c)

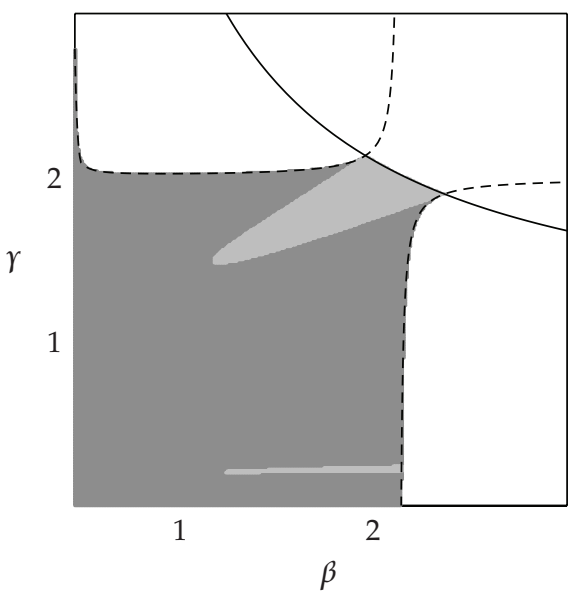

(e)

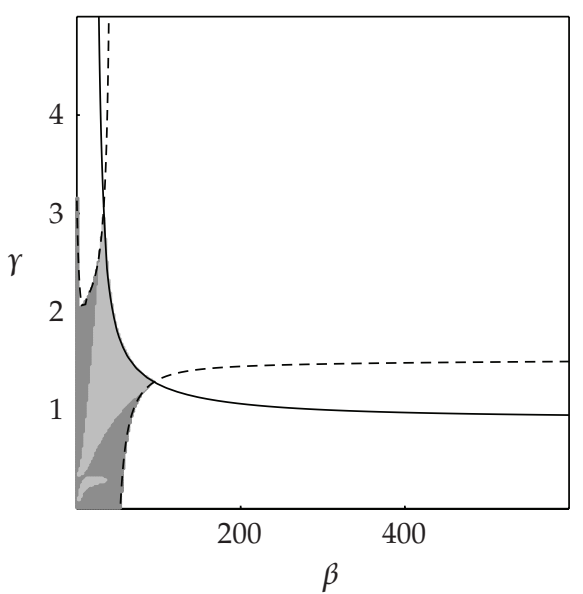

(b)

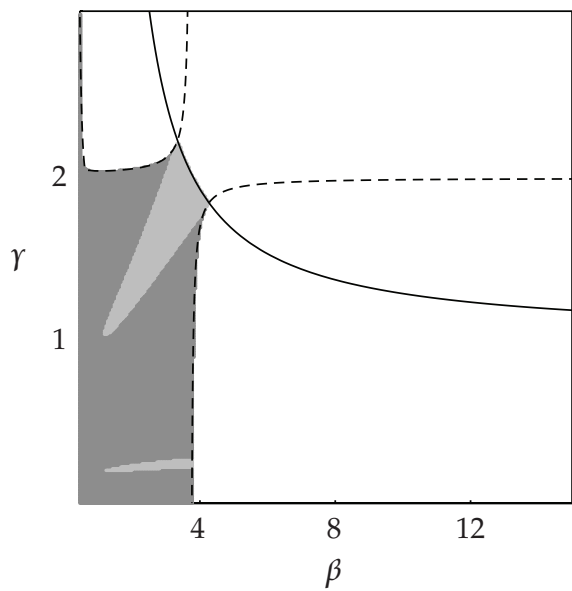

(d)

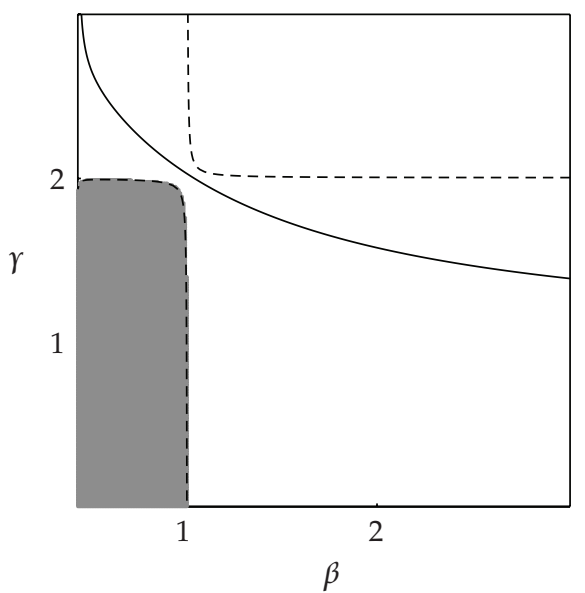

(f)

Figure 17: Stability region in the $(\beta, \gamma)$ plane for $Z=238$, and $\alpha=0.05,0.1,0.5,1,1.5,2.2$, from left to right and top to bottom (note the different scales). 


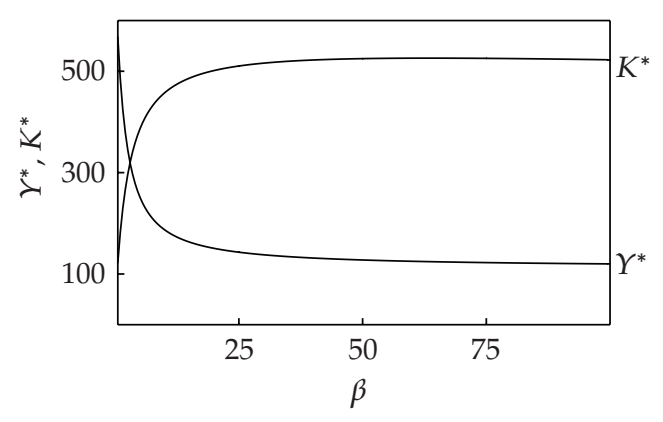

(a)

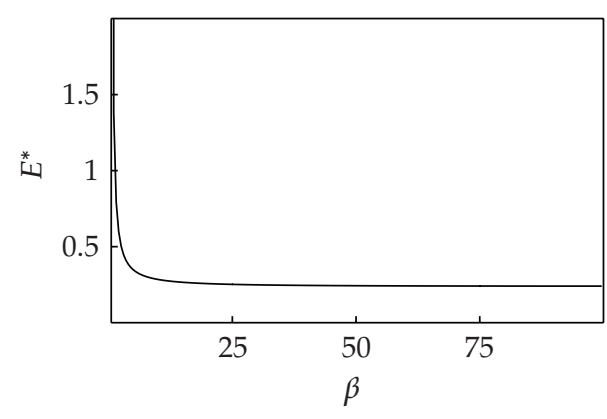

(b)

Figure 18: Equilibrium values of Model 2 as functions of $\beta$, for $Z=238$.

Equations (3.3), (8.1), and (3.2) provide the numerical values of all three variables at equilibrium for each value of $\beta$ (and given $Z$ ). Thus, for $Z=238, \beta=10$, we find $Y^{*} \cong$ $187.11, K^{*} \cong 457.51$, and $E^{*} \cong 0.284$. The equilibrium values are shown graphically as functions of $\beta$ in Figure 18 for $Z=238$. As in Model $1, Y^{*}$ (and $K^{*}$ ) is always positive for $\beta>0$. However, $E^{*}$ is positive for $\beta>0$ only when $Z<Z_{0} \cong 27$. For $Z>Z_{0}$, the value $E^{*}$ is positive only when $\beta>\beta_{\min }>0$, where $\beta_{\min }$ is the root of (3.5). For example, when $Z=238$, the minimum value of $\beta$ for which we have a positive equilibrium is $\beta_{\min } \cong 0.531$, and for $Z=50$ this minimum value is $\beta_{\min } \cong 0.086$. The relevant plot of $\beta_{\min }$ as a function of $Z$ shown in Figure 19 is produced by solving numerically the system of (3.5) and (8.1) for $Y^{*}$ and $\beta$ for each value of $Z$.

With a valid scheme for the computation of the equilibrium values of the variables, we now proceed to examine the stability of the equilibrium globally by applying the grid-search method for the determination of the stability region in a two-dimensional parameter space.

Stability in this case is determined by the roots of the characteristic polynomial of the Jacobian matrix of the two-dimensional mapping defined by the equations of model 2 for $Y$ and $E^{e}$ :

$$
\left(\begin{array}{cc}
1+\frac{\partial F_{1}^{(2)}}{\partial Y} & \frac{\partial F_{1}^{(2)}}{\partial E^{e}} \\
\frac{\partial F_{3}}{\partial Y} & 1+\frac{\partial F_{3}}{\partial E^{e}}
\end{array}\right)
$$

evaluated at the equilibrium. The characteristic equation is now quadratic:

$$
P_{2}(\lambda)=\lambda^{2}+a_{1} \lambda+a_{0}=0
$$

and the conditions for stability are

$$
1+a_{0}-\left|a_{1}\right|>0, \quad a_{0}<1
$$

The region of stability of equilibrium of Model 2, as obtained by the grid search method for $Z=238$, is shown in Figure 20 (for $\beta>\beta_{\min }$ ).

The part of the stability region in which the two roots of the characteristic equation are real is shown dark shaded, while the part in which the roots are complex conjugate is 


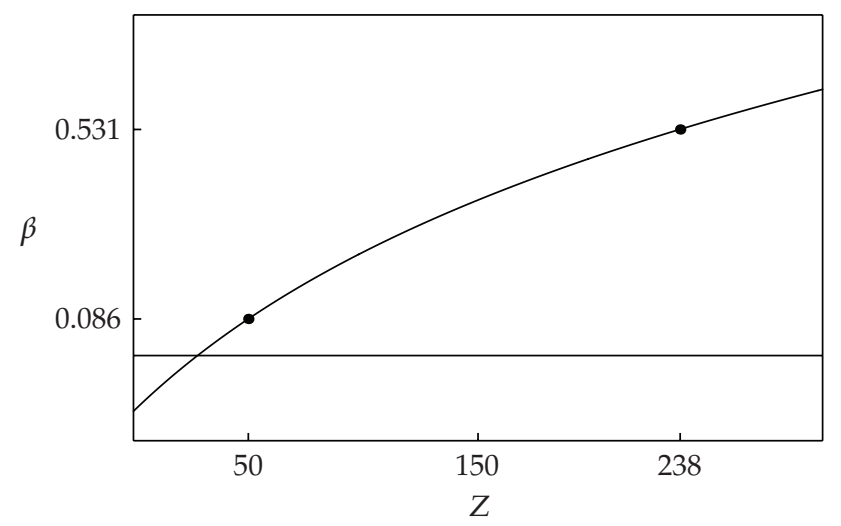

Figure 19: The minimum value of $\beta$ for positive equilibrium of Model 2, as function of $Z$.

shown light shaded. We note that in Figure 20 the first condition (8.4) is satisfied below the flip bifurcation curve:

$$
P_{2}(-1)=1+a_{0}-a_{1}=0,
$$

which is shown as a dashed curve. The second condition (8.4) is satisfied below the HopfNeimark bifurcation curve, which is shown as a continuous curve and is given in this case by

$$
a_{0}=1
$$

In the case of fixed exchange rates (Asada et al. [3]), we found that Model 2 had an entirely different dynamical behavior compared to Model 1, that is, introduction of the wealth effect in the fixed exchange rates system produced a much larger region of equilibrium stability in the parameter space and caused business cycles to disappear. We now see, by comparison of Figures 3 and 20, that in the present case of flexible exchange rates Model 2 behaves in the same way as Model 1, in so far as the behavior of the system can be determined from the formation of the stability region and the nature of its bounding curves (although Model 2 is for most values of $\beta$ destabilized somewhat faster than Model 1 when the speed of adjustment $\alpha$ is increased). In particular, as in Model 1, up to large values of the degree of capital mobility $\beta$ the bounding curve of the stability region is the flip bifurcation curve; thus, up to such large values of $\beta$ the predominant behavior of the system of Model 2 outside the stability region is period doubling and chaos, with characteristic absence of business cycles.

\section{Summary and conclusions}

We explored numerically a discrete Kaldorian macrodynamic model of an open economy with flexible exchange rates, focusing on the effects of variation of the model parameters, the speed of adjustment of the goods market $\alpha$ and the degree of capital mobility $\beta$, on the stability of equilibrium, and on the possible existence of business cycles. Using a numerical grid-search method as our main tool, we determined the stability region in the parameter space. We also identified analytically the Hopf bifurcation curve and the flip bifurcation curve, which form parts of the boundary of this region. We further computed bifurcation and 


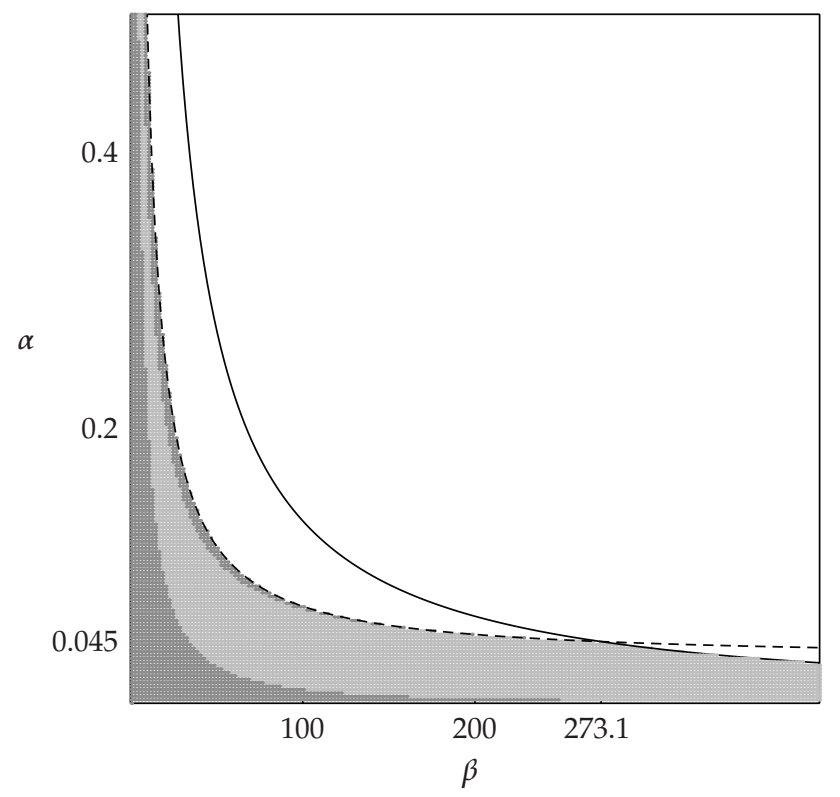

Figure 20: Region of stability of the equilibrium for Model 2 and its bounding curves in the $(\beta, \alpha)$ parameter plane for $Z=238$.

Lyapunov exponent diagrams to obtain information on the asymptotic behavior of the system when equilibrium is no longer stable, that is, outside the stability region in the parameter space.

The model exhibits complex dynamics. Certain conclusions are drawn regarding the effects of parameter changes on the stability of equilibrium and on the predominant asymptotic behavior of the system of flexible exchange rates outside the stability region. We found that at high levels of capital mobility $\beta$ stability is lost quickly, that is, at low levels of the adjustment speed. In other words, capital movement, even at very high levels, does not destabilize the system of flexible exchange rates for small values of the speed of adjustment of the goods market $\alpha$, that is, so long as firms are sufficiently prudent in their reactions, but high levels of the adjustment speed $\alpha$ are required for destabilization for relatively low levels of capital mobility $\beta$. This contrasts with the behavior of the fixed exchange rates system (see Asada et al. [3]), in which the equilibrium is destabilized at much lower levels of capital movement and/or adjustment speed. Thus, flexible exchange rates cause increased stability of equilibrium with respect to variations of the basic parameters of the model.

The predominant asymptotic behavior of the system of flexible exchange rates outside the stability region is found to be period doubling leading to chaos, and persists up to large values of the degree of capital mobility $\beta$. Examples of this dominant behavior of the system are illustrated by means of bifurcation and Lyapunov exponent diagrams. For certain extreme values of a state parameter, cycles of noticeable size and persistence were found to occur and were similarly illustrated by means of bifurcation and Lyapunov exponent diagrams, as well as diagrams of the cycles themselves in two-dimensional projections. Numerical evidence for the occurrence of subcritical bifurcations and coexistence of attractors was also provided, and examples of attractors and trajectories were presented.

We also explored briefly the effect of the parameter $\gamma$, representing the speed of adaptation of the expected rate, and found that in most cases increase of $\gamma$ destabilizes the 
system when it exceeds the value $\gamma=2$ approximately. Finally, we explored the special case (Model 2) incorporating the so-called wealth effect, which in the present case of flexible exchange rates was found to behave similarly to Model 1, contrary to the case of fixed exchange rates in which incorporation of the wealth effect causes an entirely different behavior of the system. In other words, flexibility of the exchange rates drastically reduces the significance of the wealth effect.

\section{Acknowledgment}

This work was supported by a University of Patras "K. Karatheodory" research grant.

\section{References}

[1] T. Asada, T. Misawa, and T. Inaba, "Chaotic dynamics in a flexible exchange rate system: a study of noise effects," Discrete Dynamics in Nature and Society, vol. 4, no. 4, pp. 309-317, 2000.

[2] T. Asada, T. Inaba, and T. Misawa, "A nonlinear macrodynamic model with fixed exchange rates: its dynamics and noise effects," Discrete Dynamics in Nature and Society, vol. 4, no. 4, pp. 319-331, 2000.

[3] T. Asada, C. Douskos, and P. Markellos, "Numerical exploration of Kaldorian macrodynamics: HopfNeimark bifurcations and business cycles with fixed exchange rates," Discrete Dynamics in Nature and Society, vol. 2007, Article ID 98059, 16 pages, 2007.

[4] T. Asada, "Kaldorian dynamics in an open economy," Journal of Economics, vol. 62, no. 3, pp. 239-269, 1995.

[5] N. Kaldor, “A model of the trade cycle," Economic Journal, vol. 50, no. 197, pp. 78-92, 1940.

[6] H.-W. Lorenz, Nonlinear Dynamical Economics and Chaotic Motion, Springer, Berlin, Germany, 2nd edition, 1993.

[7] G. Gandolfo, Economic Dynamics, Springer, Berlin, Germany, 1996.

[8] A. Agliari and R. Dieci, "Coexistence of attractors and homoclinic loops in a Kaldor-like business cycle model," in Business Cycle Dynamics: Models and Tools, Springer, Berlin, Germany, 2006.

[9] H. W. Lorenz, "Analytical and numerical methods in the study of nonlinear dynamical systems in Keynesian economics," in Business Cycles: Theory and Empirical Methods, W. Semmler, Ed., pp. 73-112, Kluwer Academic Publishers, Dordrecht, The Netherlands, 1994.

[10] A. Dohtani, T. Misawa, T. Inaba, M. Yokoo, and T. Owase, "Chaos, complex transients and noise: illustration with a Kaldor model," Chaos, Solitons \& Fractals, vol. 7, no. 12, pp. 2157-2174, 1996.

[11] C. Kind, "Remarks on the economic interpretation of Hopf bifurcations," Economics Letters, vol. 62, no. 2, pp. 147-154, 1999.

[12] A. Agliari, "Homoclinic connections and subcritical Neimark bifurcation in a duopoly model with adaptively adjusted productions," Chaos, Solitons \& Fractals, vol. 29, no. 3, pp. 739-755, 2006.

[13] T. Puu, Attractors, Bifurcations, and Chaos, Springer, Berlin, Germany, 2000.

[14] W. W. Chang and D. J. Smyth, "The existence and persistence of cycles in a nonlinear model: Kaldor's 1940 model re-examined," Review of Economic Studies, vol. 38, no. 1, pp. 37-44, 1971. 


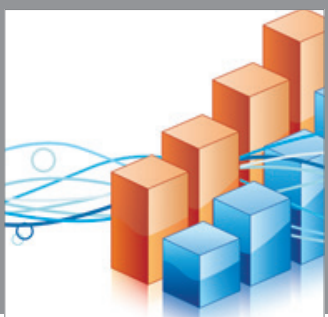

Advances in

Operations Research

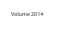

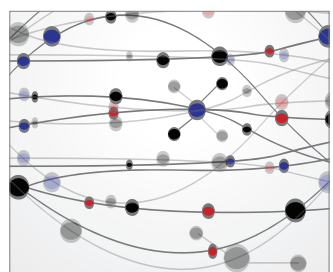

\section{The Scientific} World Journal
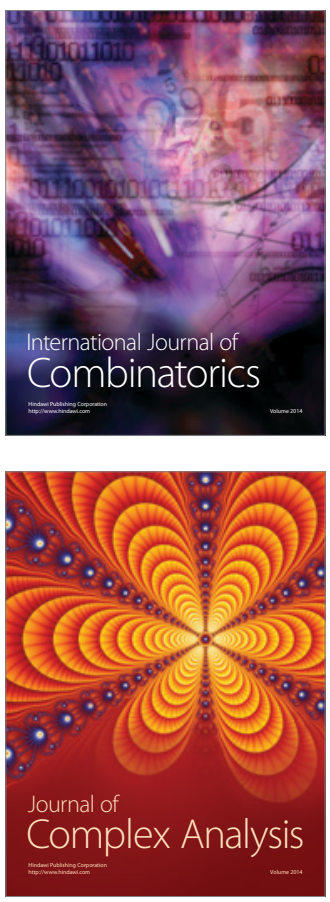

International Journal of

Mathematics and

Mathematical

Sciences
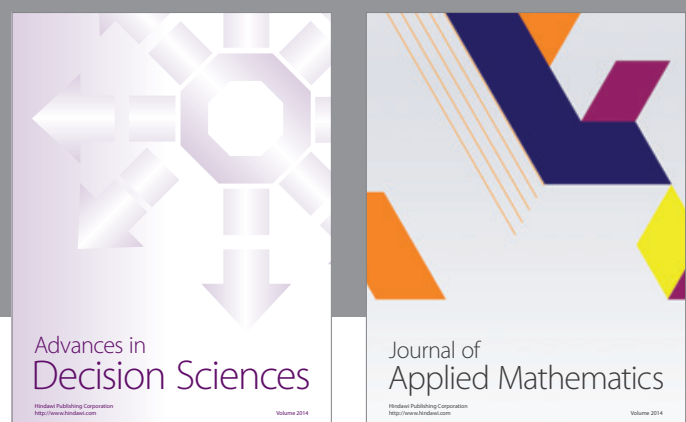

Journal of

Applied Mathematics
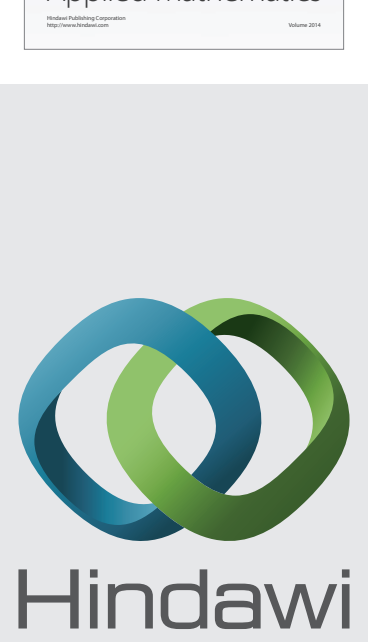

Submit your manuscripts at http://www.hindawi.com
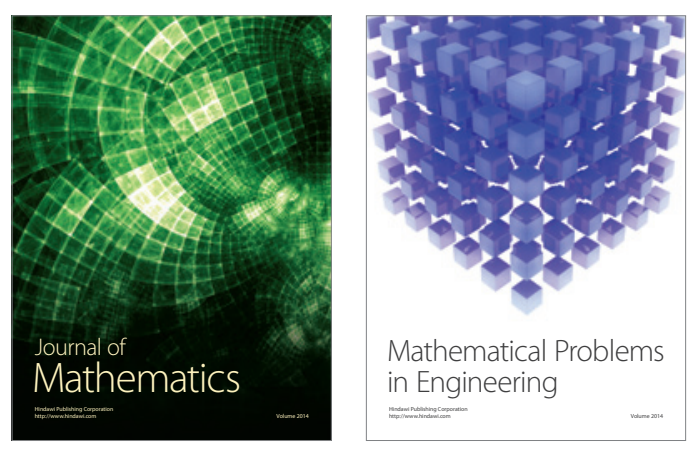

Mathematical Problems in Engineering
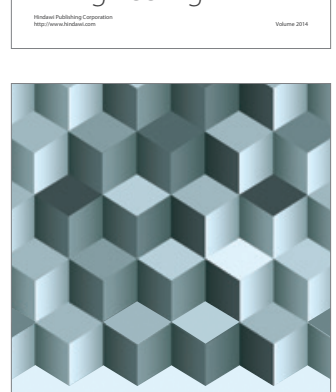

Journal of

Function Spaces
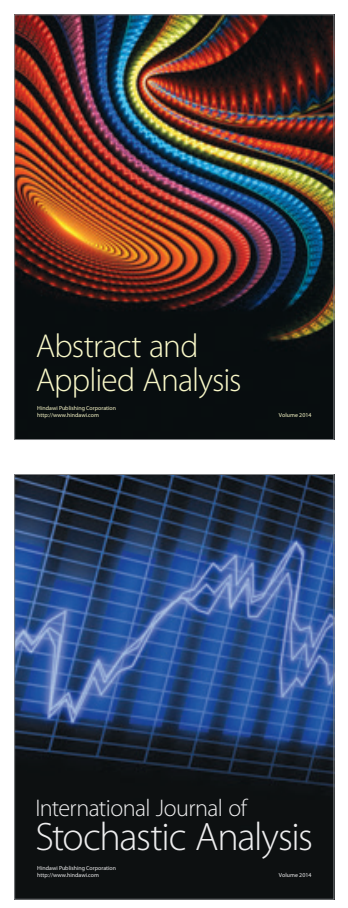

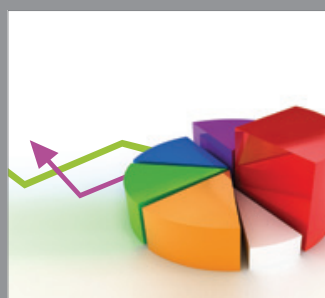

ournal of

Probability and Statistics

Promensencen
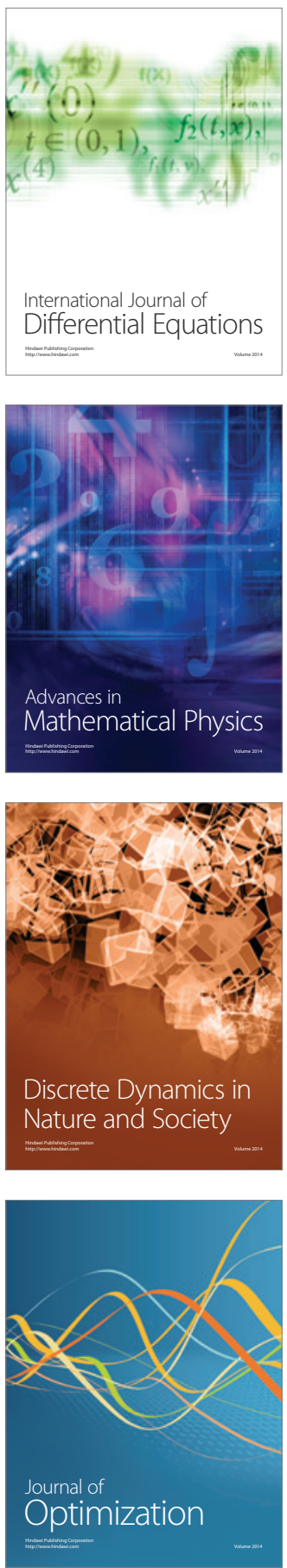